\title{
TREED GAUSSIAN PROCESS FOR MANUFACTURING IMPERFECTION IDENTIFICATION OF PULTRUDED GFRP THIN-WALLED PROFILE
}

\author{
Marco Civera $^{1,2}$, Giosuè Boscato ${ }^{3}$, Luca Zanotti Fragonara ${ }^{4}$
}

\begin{abstract}
${ }^{1}$ Ph.D. Student, Department of Mechanical and Aerospace Engineering, Politecnico di Torino, 10129 Torino, Italy. Email: $\underline{\text { marco.civera@polito.it }}$

${ }^{2}$ Visiting Research Student, Cranfield University, School of Aerospace, Transport and Manufacturing, Cranfield, United Kingdom.

${ }^{3}$ Ph.D., IUAV University of Venice, Laboratory of Strength of Materials, via Torino 153/A, 30172 Mestre, Venice, Italy. E-mail: gboscato@iuav.it

${ }^{4}$ Ph.D., Cranfield University, School of Aerospace, Transport and Manufacturing, Cranfield, United Kingdom. E-mail: 1.zanottifragonara@cranfield.ac.uk
\end{abstract}

\begin{abstract}
The process of manufacturing pultruded FRP (Fiber Reinforced Polymers) profiles involves unavoidable imperfections that affect their structural performances. This is is even more relevant for the stability of axially loaded slender elements, due to the importance of imperfections and notches to initiate the buckling phenomenon. Thus, they become a predominant factor for the design of lightweight FRP beam-like structures. A Bayesian approach is proposed to estimate the presence and location of manufacturing imperfections in pultruded GFRPs (Glass Fiber Reinforced Polymers) profiles. Specifically, the Treed Gaussian Process (TGP) procedure is applied. This approach combines regression Gaussian Processes (GP) and Bayesian-based Recursive Partitioning. The experimental and numerical modal shapes of wide flange pultruded profile were investigated. The experimental data were compared with the numerical results of several Finite Element Models (FEM) characterised by different crack sizes.
\end{abstract}

Keywords: pultruded GFRP profile, manufacturing imperfection identification, modal analysis, Treed Gaussian Process (TGP), Bayesian-based Recursive Partitioning, Finite Element Model. 


\section{INTRODUCTION}

The favourable strength-density ratio of FRP (Fiber Reinforced Polymer) material places this technology among the innovative solution in the civil engineering field. In particular, the pultruded FRP thin-walled profiles offer several applications for lightweight structures [1].

The structural performances of this technology are affected by the local and global buckling phenomena typical of thin-walled profiles. These negative aspects are emphasised by the orthotropic behaviour of FRP pultruded material, which originates from the manufacturing pultrusion process $[2,3]$. Another important aspect is that the manufacturing imperfections are sensible to the uncontrolled longitudinal distribution of fibres into profile shape during the production process.

For all these reasons, the assessment of manufacturing imperfections and of their effects is an unavoidable step for the performance characterisation of pultruded material. Generally, the unpredicted defects can affect the integrity and the stability of structures: for instance, they can trigger local failure induced by fiber buckling and resin breakage due to the non-uniform distribution of stresses on cross-section shape.

However, even if the formation of initial imperfections cannot be avoided in the manufacturing process, taking into account the effect of these imperfections still allows the designer to have an optimised design approach to reduce their deleterious effects in serviceability conditions [4].

The deviation from the idealised geometric and mechanical configuration, due to manufacturingprocess imperfection, triggers and increments the discrepancy between the analytical prediction and experimental results above all for the buckling phenomena. Tuning with this topic, many researches have been carried out on composite cylindrical shell affected by geometric imperfections [5-7]. Recently, the buckling phenomenon for composite cylindrical shell has been analysed considering both geometric imperfections and embedded delamination imperfections to define the lowerbound buckling loads [8]. The so-called stochastic method has been also proposed to assess the imperfection sensitive of composite cylinders [9]. The work of Boscato and Ientile [10] aims at defining the effects of geometrical and mechanical imperfection on buckling phenomena through the application of Vibration-Based Non-Destructive Testing (NDT) on pultruded GFRP columns. Similarly to this latter research, experimental modal analysis has been used in this paper to characterise the pultruded GFRP profile assuming that the mechanical, geometric and physical anomalies may affect the modal parameters.

NDT damage identification procedures present several advantages with respect to other testing procedures. They can be classified as local or global [11]. Local methods, such as e.g. ultrasonic 
testing and X-ray methods, are very common approach; however, thay are slow to perform throughout large elements. Vibrational-based techniques allow overcoming these limits with a global approach. These last methods are based on changes in mechanical and physical characteristics involving the variation of dynamic parameters (frequencies, mode shapes and damping ratios). In particular, the vibration-based damage identification can be carried out by methods based on frequency, mode shape, curvature mode shape and methods using both frequency and mode shape [12-14]. Vibration-based methods are particularly suitable for slender monodimensional structures with small cracks and/or imperfections. Natural frequency-based techniques are commonly used for damage detection and localisation [15]. However, the frequency shifts can be compromitted by environmental and noise conditions and it is not well-suited for multi-damage identification. In fact, the problem of estimating the crack properties (severity and position) from the changes in their natural frequencies may become ill-posed [16] and thus not reliable. On the other hand, mode shape-based methods are more apt for damage localisation, thanks to their higher spatial resolution. The environmental effects are negligible whereas the noise contaminations and the number of sensors are significative. The effectiveness of mode shape-based approaches has been validated, for instance, in the framework of Extreme Function Theory [17] and from video-acquired data [18].

Nevertheless, some limitations still affect these methods, such as the relatively low sensitivity to damage and the interpretability of the results, which requires an expert user. Hence, a principled and automated damage-localisation algorithm would help in reducing the possibility of human error; the TGP routine is proposed in this sense. Importantly, deriving twice the eigenvectors can be useful to better highlight the damage- or imperfection-induced discontinuities, but at the cost of increasing the unrelated effects of measurement noise, further reducing the interpretability of the results. Thus, on a case-by-case basis, it may be more or less convenient to consider the modal curvatures rather than the 'raw' mode shapes. Both approaches has been tested here.

The TGP algorithm has been already successfully applied in signal processing for SHM purposes, noteworthy on response surface models [19] and on the time-domain, proving its usefulness especially for heteroskedastic data (i.e., with time-varying statistical properties) [20; 21]. The algorithm has been recently applied on 1-dimensional beam elements for damage detection by numerical analysis [22].

The potential of the TGP method for the assessment of manufacturing imperfections is investigated here. A wide flange GFRP pultruded profile that showed anomalies in flexural modal shapes has been used as a case study. The TGP method has been applied on several Finite Element models and 
on experimental data; promising results were achieved. The rest of this paper is organised as follow. Section 2 provides an overview of the TGP and of its intended use in the context of this research. Section 3 describes the experimental investigation of the case study and the FE models used for the preliminary analysis. Section 4 reports the results for both the numerical and the experimental cases. The paper ends with the Conclusions.

\section{OVERVIEW ON TREED GAUSSIAN PROCESSES FOR THE IDENTIFICATION OF ANOMALIES.}

\subsection{Gaussian Process (GP) Regression}

Gaussian Process (GP) Regression is nowadays a fairly established Machine Learning (ML) approach for surrogate modelling in the context of Structural Health Monitoring (SHM). Firstly envisioned by Rasmussen and Williams [23], the procedure has gained the attention of the SHM community since at least one decade [24] and has been intensely applied, especially for the metamodeling of time series.

The key concept is quite straightforward as it is a Gaussian distribution over functions rather than over scalar values. The most important consequence is that, differently from the large majority of the regression models which only return a defined $f(\boldsymbol{x})$ for any given $\boldsymbol{x}$ ( $\boldsymbol{x}$ being, in this case, a 1dimensional array), the GP Regression naturally returns a Gaussian probability distribution function (pdf). This implies that the mean value can be utilised as the best estimate of the function $f(x)$ for any term included into $\boldsymbol{x}$ (which can be an instantaneous value in recorded time histories, an output channel in a sensor network, or whatever input can be defined in a vectorial form). Even more importantly, the whole approach is Bayesian principled and it is then possible to use the function standard deviation to define automatically a confidence interval (CI) around its predicted values.

In a Bayesian sense, it is possible to define the prior beliefs about the corresponding outputs as a multivariate normal distribution. Therefore, for a given set of $n$ input points, such that $\left\{\boldsymbol{x}_{1}, \boldsymbol{x}_{2}, \ldots, \boldsymbol{x}_{n}\right\}$ with $\boldsymbol{x}_{i} \in \square^{1 \times d} \forall i$, the mean function of this multivariate normal distribution can be seen as least-squares regression fit through $\boldsymbol{x}$, i.e.

$$
E[f(\boldsymbol{x}) \mid \boldsymbol{\beta}]=m(\boldsymbol{x})=\boldsymbol{h}(\boldsymbol{x})^{T} \boldsymbol{\beta}
$$

Where $\boldsymbol{\beta}$ is the vector of coefficients corresponding to the regression function $\boldsymbol{h}(\boldsymbol{x})$. Since the prior beliefs can be quite arbitrarily set, a linear regression can be imposed for $\boldsymbol{h}(\boldsymbol{x})$, or it may even be set to zero. A zero-mean prior will be used for simplicity. It is then possible to (again, quite 
arbitrarily) define the (constant) hyperparameters that will define the covariance between output points, that is to say, the scaling factor $\sigma_{f}^{2}$, the noise variance $\sigma_{n}^{2}$, and the diagonal matrix of inverse length-scales $L$, such that

\begin{tabular}{|c|c|}
\hline $\operatorname{cov}\left[f(\boldsymbol{x}), f\left(\boldsymbol{x}^{\prime}\right) \mid \sigma_{f}^{2}, \sigma_{n}^{2}, L\right]=k\left(\boldsymbol{x}, \boldsymbol{x}^{\prime}\right)=\sigma_{f}^{2} \exp \left[-\left(\boldsymbol{x}-\boldsymbol{x}^{\prime}\right)^{T} L\left(\boldsymbol{x}-\boldsymbol{x}^{\prime}\right)\right]+\sigma_{n}^{2} \delta$ & 2 \\
\hline
\end{tabular}

Where $k\left(\boldsymbol{x}, \boldsymbol{x}^{\prime}\right)$ is a shorthand for any valid covariance function; one of the one most often used is the squared-exponential (SE) function reported above (note that $\delta=\left\{\begin{array}{ll}1 & \text { if } \boldsymbol{x}=\boldsymbol{x}^{\prime} \\ 0 & \text { otherwise }\end{array}\right.$ ).

Thus, with the set of prior beliefs and the chosen covariance function, the prior definition of the problem is complete. The posterior distribution can be simply achieved by conditioning this prior distribution on functions with the training data (i.e. the vector of output points corresponding to the input training set). Thanks to the marginalisation properties of the (multivariate) Gaussian density, it is possible to consider the function $f(\boldsymbol{x})$ at a finite number of points of interest. Considering $\boldsymbol{f}$ as the ensemble of the function values corresponding to the training points (these latter ones arranged into a matrix $\boldsymbol{X}$ of training vectors) and $f^{*}=f\left(\boldsymbol{x}^{*}\right)$ as the predicted function value corresponding to the new point $x^{*}$, these can be defined as a Gaussian distribution with characteristics

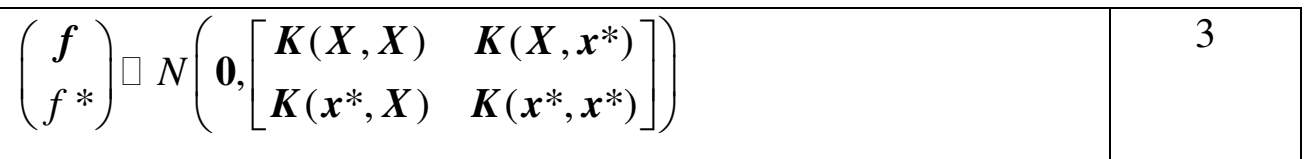

For a zero-mean prior as specified before and where $\boldsymbol{K}(\boldsymbol{X}, \boldsymbol{X}), \boldsymbol{K}\left(\boldsymbol{X}, \boldsymbol{x}^{*}\right)$, and $\boldsymbol{K}\left(\boldsymbol{x}^{*}, \boldsymbol{X}\right)$ are, respectively, a matrix made up by $k\left(\boldsymbol{x}_{i}, \boldsymbol{x}_{j}\right)$ for any $i$-th and any $j$-th term, a column vector made up by $k\left(\boldsymbol{x}_{i}, \boldsymbol{x}^{*}\right) \forall i$, and the transpose of the latter. By assuming a Gaussian noise model for the observed target data

$$
\boldsymbol{y} \square N\left(\boldsymbol{f}, \sigma_{n}^{2} \boldsymbol{I}\right)
$$

With the noise variance multiplying the identity matrix $\boldsymbol{I}$. It is then possible to marginalise $\boldsymbol{f}$ from Eq 3, resulting in a joint distribution for the testing and training target values,

$$
\left(\begin{array}{c}
\boldsymbol{y} \\
y^{*}
\end{array}\right) \square N\left(\mathbf{0},\left[\begin{array}{cc}
\boldsymbol{K}(\boldsymbol{X}, \boldsymbol{X})+\sigma_{n}^{2} \boldsymbol{I} & \boldsymbol{K}\left(\boldsymbol{X}, \boldsymbol{x}^{*}\right) \\
\boldsymbol{K}\left(\boldsymbol{x}^{*}, \boldsymbol{X}\right) & \boldsymbol{K}\left(\boldsymbol{x}^{*}, \boldsymbol{x}^{*}\right)+\sigma_{n}^{2}
\end{array}\right]\right)
$$

By re-arranging the joint probability distribution into the form of a conditional distribution, one obtains the posterior distribution for the unknown scalar $y^{*}$,

$$
y^{*} \square N\left(m^{*}\left(\boldsymbol{x}^{*}\right), k^{*}\left(\boldsymbol{x}^{*}, \boldsymbol{x}^{*}\right)\right)
$$

With the posterior mean of the GP Regression 


$$
m^{*}\left(\boldsymbol{x}^{*}\right)=k^{*}\left(\boldsymbol{x}^{*}, \boldsymbol{X}\right)\left[\boldsymbol{K}(\boldsymbol{X}, \boldsymbol{X})+\sigma_{n}^{2} \boldsymbol{I}\right]^{-1} \boldsymbol{y}
$$

And the posterior variance of the same

$k^{*}\left(\boldsymbol{x}^{*}, \boldsymbol{x}^{\prime}\right)=k\left(\boldsymbol{x}^{*}, \boldsymbol{x}^{\prime}\right)-\boldsymbol{K}\left(\boldsymbol{x}^{*}, \boldsymbol{X}\right)\left[\boldsymbol{K}(\boldsymbol{X}, \boldsymbol{X})+\sigma_{n}^{2} \boldsymbol{I}\right]^{-1} \boldsymbol{K}\left(\boldsymbol{X}, \boldsymbol{x}^{\prime}\right)$

Which can act, respectively, as the pointwise best estimate of the regression problem and its related confidence interval. To conclude, it is possible to determine the optimal hyperparameters by maximising the log marginal likelihood

$$
f(\boldsymbol{\theta})=\frac{1}{2} \boldsymbol{y}^{T}\left[\boldsymbol{K}(\boldsymbol{X}, \boldsymbol{X})+\sigma_{n}^{2} \boldsymbol{I}\right] \boldsymbol{y}-\frac{1}{2} \log \left[\boldsymbol{K}(\boldsymbol{X}, \boldsymbol{X})+\sigma_{n}^{2} \boldsymbol{I}\right]
$$

Being $\boldsymbol{\theta}$ the complete set of these parameters, which generally is very small dimensionally, thus allowing to be simply achievable with gradient descent. This way, a single covariance kernel is defined above all the input space. This implicitly assumes that the structure is uniform in space (or constant in time, depending on the kind of input), as the same hyperparameters apply everywhere / at any instant. In a spatial domain, this assumption of uniform smoothness does not allow to model crack or defects as pointwise discontinuities - these are simply "smoothed" away by the regression. These short-scale spatial discontinuities require therefore a pointwise regression strategy to be identified and modelled. This requires some sort of "switching point" above neighbouring regions; several GP-based approaches have been proposed in this sense for damage localisation in beams $[25 ; 26]$. The approach followed here resort to Treed Gaussian Processes (TGPs).

\subsection{Treed Gaussian Processes (TGPs).}

In the most concise definition possible, the TGP methodology combines GP Regression with a Bayesian Classification and Regression Tree (CART) framework. The basic theory behind CARTs and their Bayesian extension can be found, respectively, in [27-29]. However, the Chipman original formulation only relied on linear regression models. The currently-used TGP approach stems from these field of research, as it is well-described in Gramacy's PhD thesis [30], and include separate GPs ar regression models within the branches of the tree.

Due to the complexity of the algorithm, the interested audience is invited to refer directly to [30] for a detailed description. To make this discussion self-concluded, an (approximated) algorithm flowchart is reported here in Figure 1. For the Randomised Tree Alteration step, four jumping criteria were defined in the Markov space - Grow, Prune, Change, and Rotate -accordingly to their original implementation by Chipman [28]. 
The aim is to define a maximum a posteriori (MAP) tree $\hat{T}$ and its parameters $\hat{\theta} \mid \hat{T}$. The main difficulty lies in the incorporation of the GP Regression algorithm into a Bayesian regression tree formulation. The concept is, therefore, to remove both $\beta$ and $\sigma_{f}^{2}$ from the optimisation problem, by marginalising over them. The analytical formulations were summarised in [22], and a much more detailed discussion is available in [31]. Although the expressions are closed-form, they become quite complex and computationally expensive to be solved. This derives from the problem being reduced to the form of a Student t-process, conditional on the training data and $L$, i.e.

$$
[f(\boldsymbol{x}) \mid y, L] \square t_{n-q}\left(m^{*}(\boldsymbol{x}), \hat{\sigma}_{f}^{2} k^{*}(\boldsymbol{x}, \boldsymbol{x})\right)
$$

Where the updated scaling factor $\hat{\sigma}_{f}^{2}$ derives from the marginalisation procedure, and so does the updated vector of parameters $\hat{\boldsymbol{\beta}}$ from which $m^{*}(\boldsymbol{x})$ can be analyticallí define. Both $\hat{\sigma}_{f}^{2}$ and $\hat{\boldsymbol{\beta}}$ can be proved to coincide with their least-square estimates; the technical difficulties arise from $L$. The diagonal matrix of roughness parameters $L$ cannot be analytically integrated out. Henceforth, a Markov Chain Monte Carlo (MCMC) random walk procedure has to be applied. This is by far the most power-demanding and time-consuming part of the whole algorithm but there is no easy shortcut to bypass it, at least for the time being.

Consider that in the actual Gramacy implementation of the TGP algorithm [32], the Bayesian procedure is further complicated since all hyperparameters (including $L$ ) are further represented by prior densities and by their hyper-hyperparameters, hierarchically.

To conclude, a GP regressor (defined as in the previous Subsection 2.1) is fitted on each independent region. These, in turn, are defined as partitions of the input space via a Bayesian CART.

Since each GP regressor exists in a well-defined (1-dimensional) spatial region, the branching points between contiguous leaves of the MAP tree would ideally be located in correspondence with discontinuities of the provided mode shapes. For a uniform, uncracked beam, these points are the most likely defect positions.

Among the many critical points of the procedure, two need to be taken in particular consideration:

1. The Bayesian procedure is highly dependent on the number and the distribution of the training data points (as in almost any ML application); thus the resulting partitioned model will reflect the quantity, quality, and spatial distribution of the input data.

2. The acceptance criterion applied in the MCMC process is essential to abort or validate the new walked-in tree state at any subsequent step; this point was (here and in the previous 
applications) solved by resorting to a Metropolis-Hastings ratio, computed as the posterior logprobability of the attempted new tree over the one from the current one defined at the previous step. This criterion discriminates between updating or not the current tree and its parameters, as it can be seen in Figure 1. The threshold for acceptance was required to be inferior to a uniformly sampled random value included between $[0,1]$.

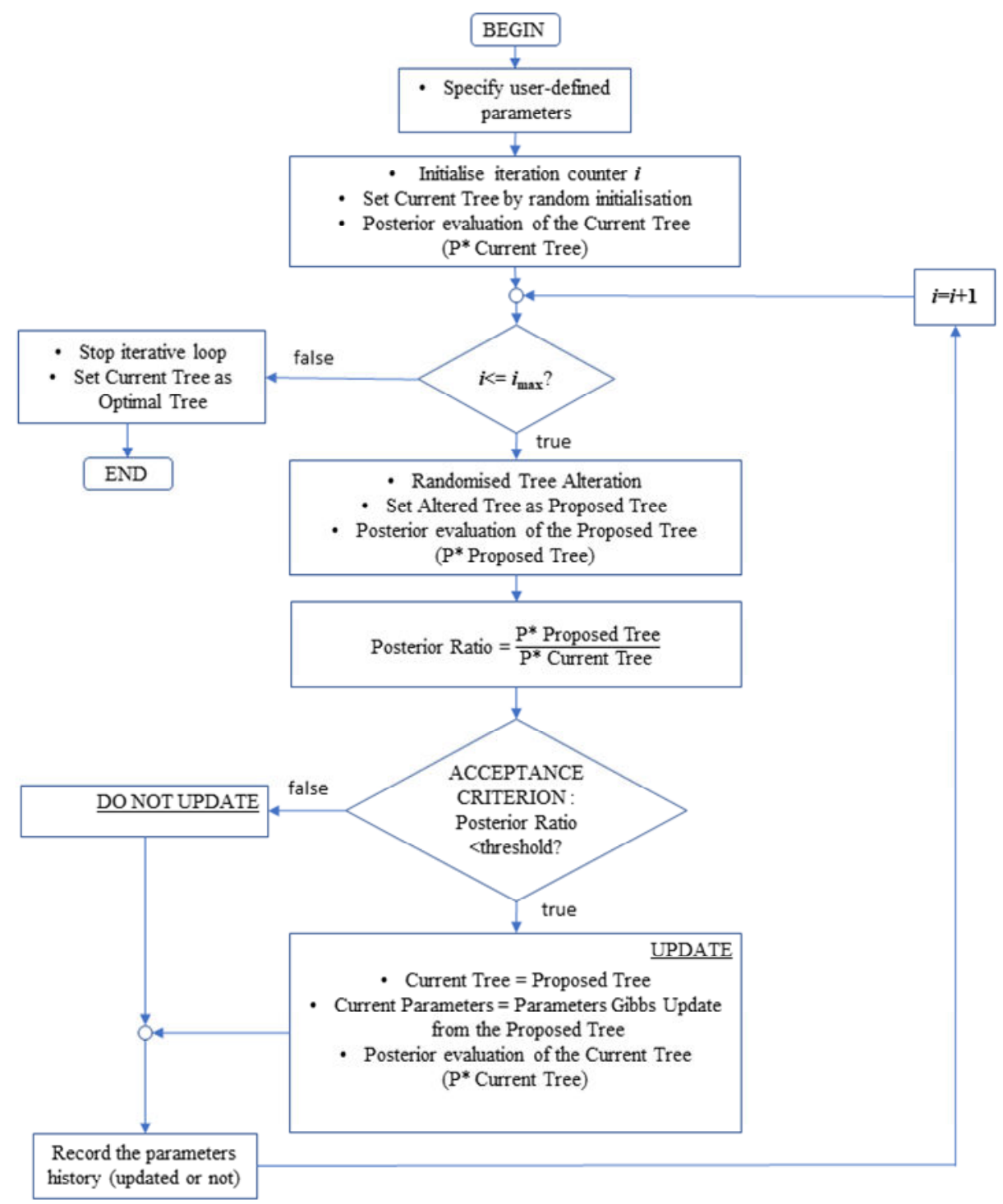

Figure 1. Illustrative flowchart of the Treed Gaussian Process algorithm.

\section{3 the implemented code.}

All computations were performed via an $R$ script which implements the TGP package proposed by Gramacy in [32]. The function btgpllm() was used, allowing jumps to the limiting linear model (LLM) whenever (and if) possible, to potentially exploit any region of the input space with zero or 
quasi-zero response to speedup the partitioning procedure. Following the best practices described in [29], the Markov Chain has been restarted to re-explore the search space and storing only the most fitted result. The MCMC procedure was initialised with an additional run of a Bayesian treed linear model (linear burn-in, with a pre-split of the input space), followed by a second burn-in period of 2000 rounds to define a stationary Markov Chain and then 5000 rounds to establish the statistics. At each restart, $\hat{T}$ and its corresponding $\hat{\theta} \mid \hat{T}$ were stored, including the branching points.

\section{EXPERIMENTAL AND NUMERICAL MODAL ANALYSIS}

\subsection{Case study: GFRP pultruded profiles}

The structural performances of pultruded GFRP thin-walled profiles are characterised by orthotropic properties and elastic-brittle behaviour. The pultrusion manufacturing process determines a strong axis along the fibres that coincides with the main dimension of profile and the weak transversal axes that define the cross-section plane [33; 34]. In particular, the pultruded material is composed by three parts: i) the internal unidirectional roving made by E-Glass longitudinal fibre reinforcement arranged along the main axis of the profile; ii) the external continuous mat made by E-Glass short fibre reinforcement distributed on triaxial layers with $+/-45^{\circ}$ and $0^{\circ}$; iii) the continuous phase constituted by thermoset Vinilester 980-35 resin-based matrix. The percentage of fibres is $45 \%$ of the volume.

Table 1 reports the mechanical properties of pultruded profile determined using coupons extracted from a specimen of profile and tested in accordance with the relevant standards [35-38].

An open wide flange cross-section with simply supported boundary condition was experimentally investigated here (Figure 2). The profile reference system is shown in Figure 2 with the z-axis parallel to the main dimension of profile and normal to xy plan that defines the cross-section. The geometrical characteristics of the wide flange profile are summarized in Table 2.

\begin{tabular}{lcc}
\hline Properties & Notation & Mean value \\
\hline Longitudinal tensile modulus of elasticity & $E_{z}=E_{L}$ & $28.5(\mathrm{GPa})$ \\
Transverse tensile modulus of elasticity & $E_{y}=E_{x}=E_{T}$ & $8.5(\mathrm{Gpa})$ \\
Transverse shear modulus of elasticity & $G_{y x}=G_{L}$ & $3.5(\mathrm{Gpa})$ \\
In-plane shear modulus of elasticity & $G_{x z}=G_{y z}=G_{T}$ & $2.5(\mathrm{Mpa})$ \\
Longitudinal Poisson's ratio & $v_{z x}=v_{z y}=v_{L}$ & 0.25 \\
Transversal Poisson's ratio & $v_{x y}=v_{y x}=v_{T}$ & 0.12 \\
Longitudinal tensile strength & $\sigma_{z t}=\sigma_{L t}$ & $350(\mathrm{Mpa})$ \\
Transverse tensile strength & $\sigma_{x t}=\sigma_{y t}=\sigma_{T t}$ & $70(\mathrm{Mpa})$ \\
Longitudinal compressive strength & $\sigma_{z c}=\sigma_{L c}$ & $413(\mathrm{Mpa})$ \\
Transverse compressive strength & $\sigma_{x c}=\sigma_{y c}=\sigma_{T c}$ & $80(\mathrm{Mpa})$ \\
Shear strength & $\tau_{x y}=\tau_{x z}=\tau_{y z}$ & $40(\mathrm{Mpa})$ \\
\hline & 9 &
\end{tabular}


Table 1. Mechanical properties of pultruded material.

It is possible to discern the different stiffness through the maximum $\left(\mathbf{J}_{\max }\right)$ and minimum $\left(\mathbf{J}_{\min }\right)$ moment of inertia, better captured by the accelerometer positioned along $\mathrm{y}$ - and $\mathrm{x}$-axes respectively (Figure 2).
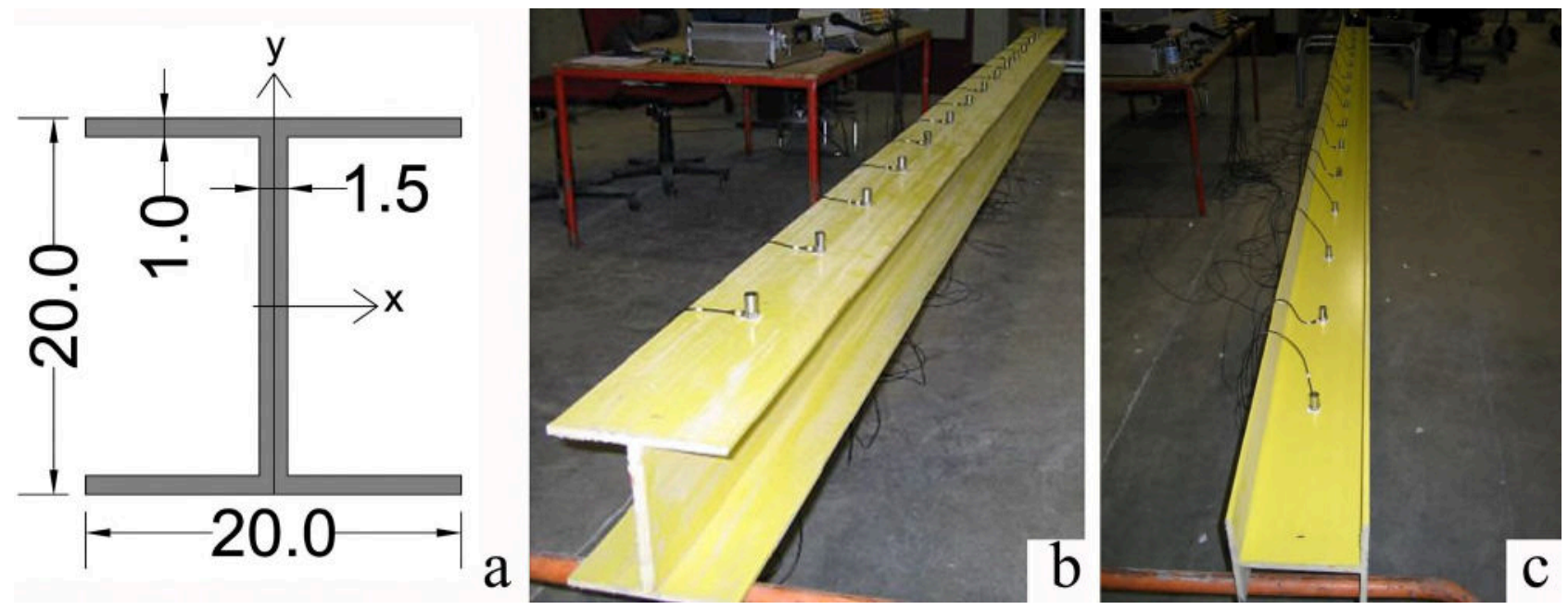

Figure 2. Wide flanges profile, geometrical details for the cross-sectional shape (a) (dimensions in centimetres), photos along y (b) and $\mathrm{x}(\mathrm{c})$ axes.

\begin{tabular}{|c|c|c|c|c|c|c|}
\hline Profile & $\begin{array}{c}\text { Second moment } \\
\text { of area } \\
J\left(\mathrm{~cm}^{4}\right)\end{array}$ & $\begin{array}{c}\text { Torsional } \\
\text { moment of area } \\
J_{t}\left(\mathrm{~cm}^{4}\right)\end{array}$ & $\begin{array}{l}\text { Length } \\
L(\mathrm{~cm})\end{array}$ & Area A $\left(\mathrm{cm}^{2}\right)$ & $\begin{array}{l}\text { Weight } \\
\text { (kg) }\end{array}$ & $\begin{array}{l}\text { Density } \\
\left(\mathrm{kg} / \mathrm{m}^{3}\right)\end{array}$ \\
\hline $\begin{array}{l}\text { "WF", } \mathrm{J}_{\max } \\
\text { "WF", } \mathrm{J}_{\text {min }}\end{array}$ & $\begin{array}{r}4342.3 \\
1338.4 \\
\end{array}$ & 33.58 & 500 & 62 & 57.35 & 1850 \\
\hline
\end{tabular}

Table 2. Geometrical and physical properties of profiles

\subsection{Experimental Modal Analysis}

Regarding the experimental setup, the simply supported condition was achieved by positioning the beam on cylindrical elements with a span of $4.9 \mathrm{~m}$. The excitation points (from B0 to B15) and the accelerometer positions (from A1 to A15) were defined as portrayed in Figure 3; they are shown for half beam considering a grid-step by $153 \mathrm{~mm}$. Figure 3 has been subdivided according to the two testing configurations, i.e. along with $\mathrm{x}$ - and $\mathrm{y}$-axes, reported in the first and second row respectively.

The 15 excitation points were given by an instrumented hammer (Dytran 5850A) synchronized with 15 accelerometers (BBN model 507Lf) for the recording phase. All recorded signals were post-processed by a digital bandpass filter with a range of $1 \mathrm{~Hz}-150 \mathrm{~Hz}$. 


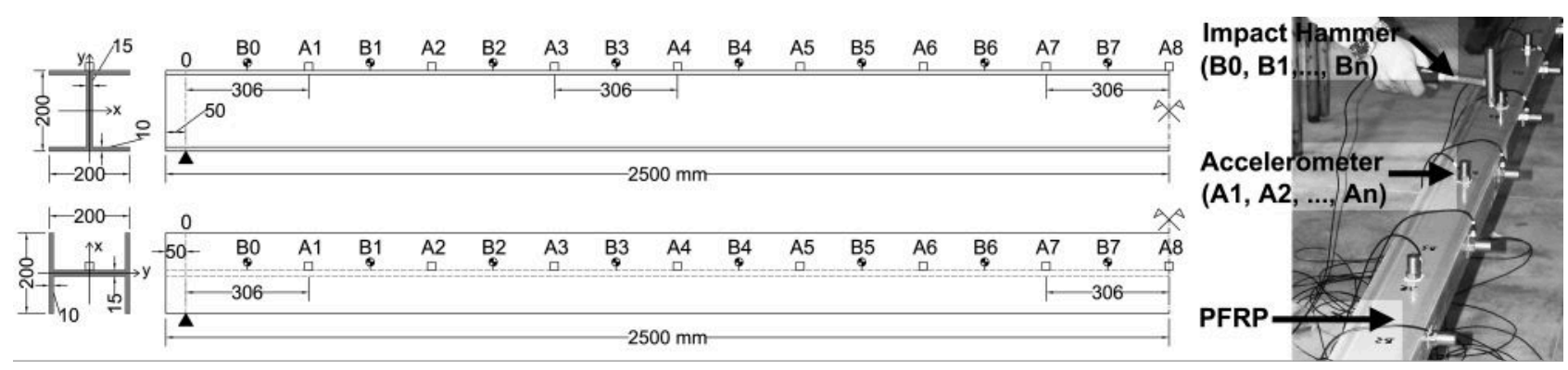

Figure 3. Test setup

The Eigensystem Realization Algorithm (ERA) [39] has been applied to define the stabilisation diagrams. Figure 4 shows an example for one set of acquired data. The main dynamic parameters can be identified by stable modes highlighted by filled circles considering the following limits: frequency variation $\leq 1 \%$; damping variation $\leq 10 \%$; minimum Modal Assurance Criterion [40] $>$ 0.95 . 


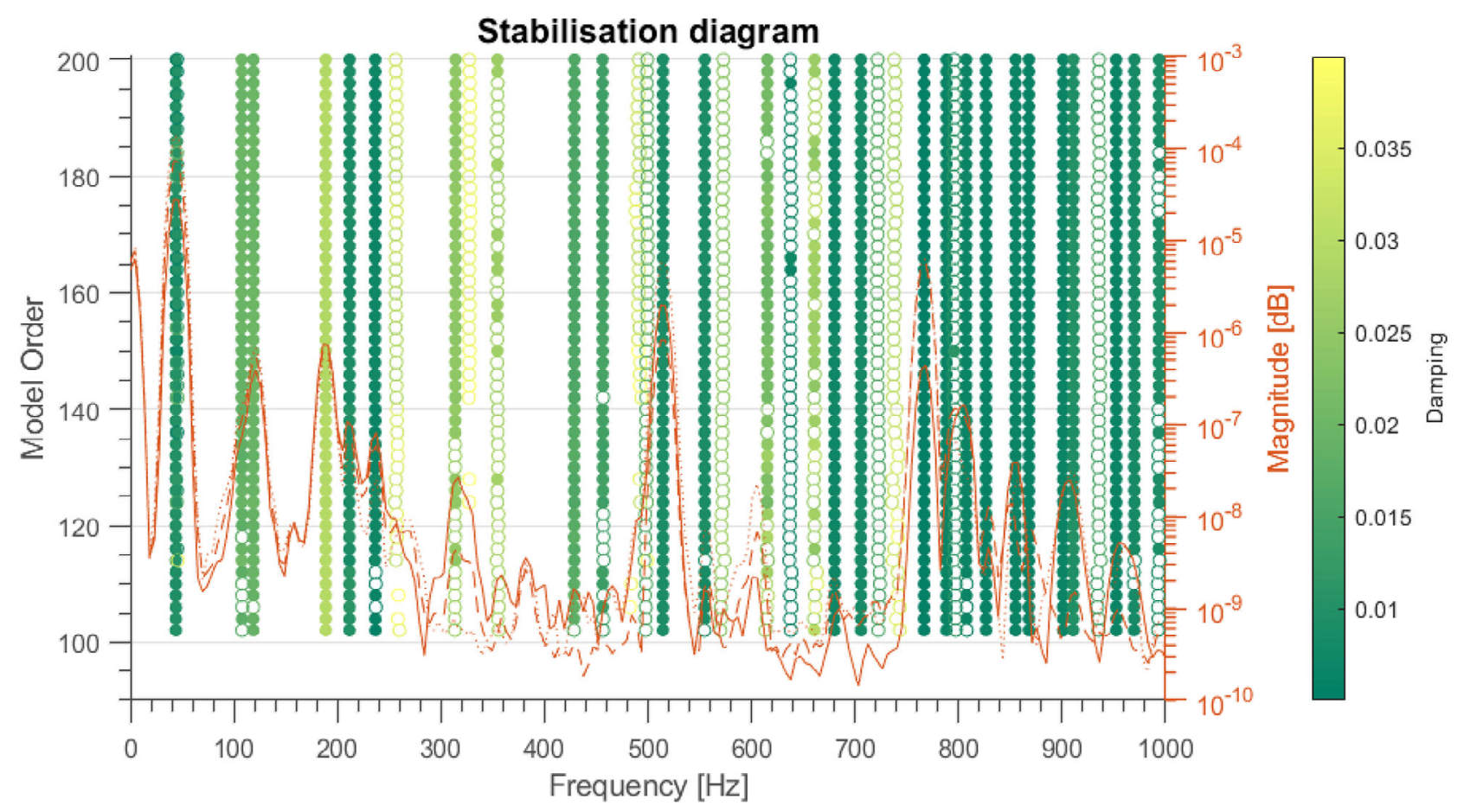

(a)

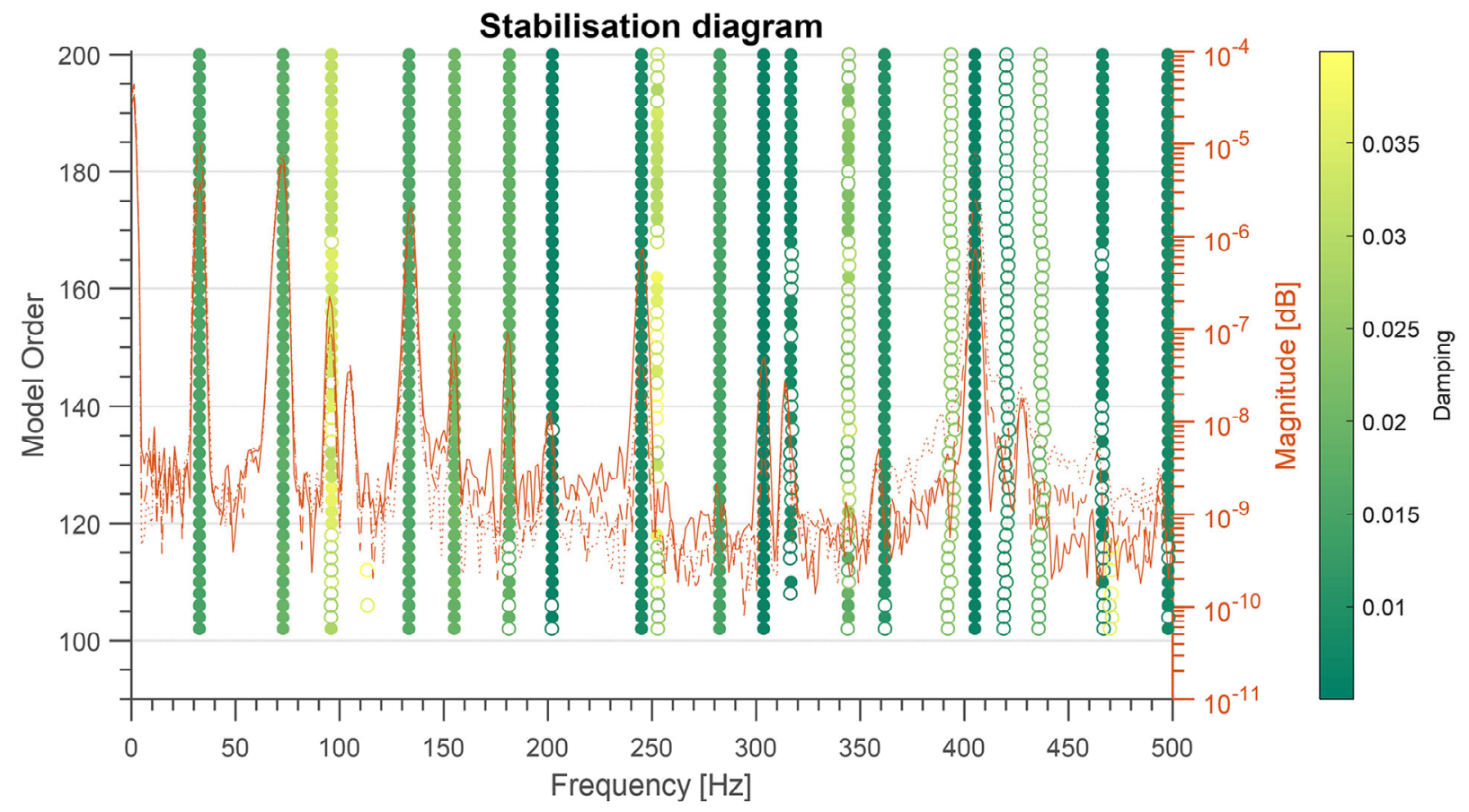

(b)

Figure 4: Stabilisation diagrams: (a) x-direction channels and (b) y-direction channels using the B3 excitation position. 
The results of the experimental modal analysis are listed in Table 3.

\begin{tabular}{cccc}
\hline Modal Shapes & Classification & Frequency [Hz] & Damping [\%] \\
\hline 1 & $1^{\text {st }} \mathrm{y}$ & 32.51 & 1.48 \\
2 & $1^{\text {st }} \mathrm{x}$ & 43.95 & 1.04 \\
3 & $2^{\text {nd }} \mathrm{x}$ & 118.77 & 2.66 \\
4 & $2^{\text {nd }} \mathrm{y}$ & 133.18 & 1.81 \\
5 & $3^{\text {rd }} \mathrm{y}$ & 180.55 & 1.14 \\
6 & $3^{\text {rd }} \mathrm{x}$ & 188.20 & 2.61 \\
7 & $4^{\text {th }} \mathrm{x}$ & 233.32 & 1.40 \\
8 & $4^{\text {th }} \mathrm{y}$ & 244.61 & 0.95 \\
\hline
\end{tabular}

Table 3. Experimental modal analysis results.

All modal shapes identified by each impact are shown in Figure 5. The most evident feature is the non-smoothness and lack of symmetry of the mode shapes, due probably to the geometrical and mechanical imperfections of the profile. The dispersion of the mode shape for each impact is very low for the profile with the maximum moment of inertia.
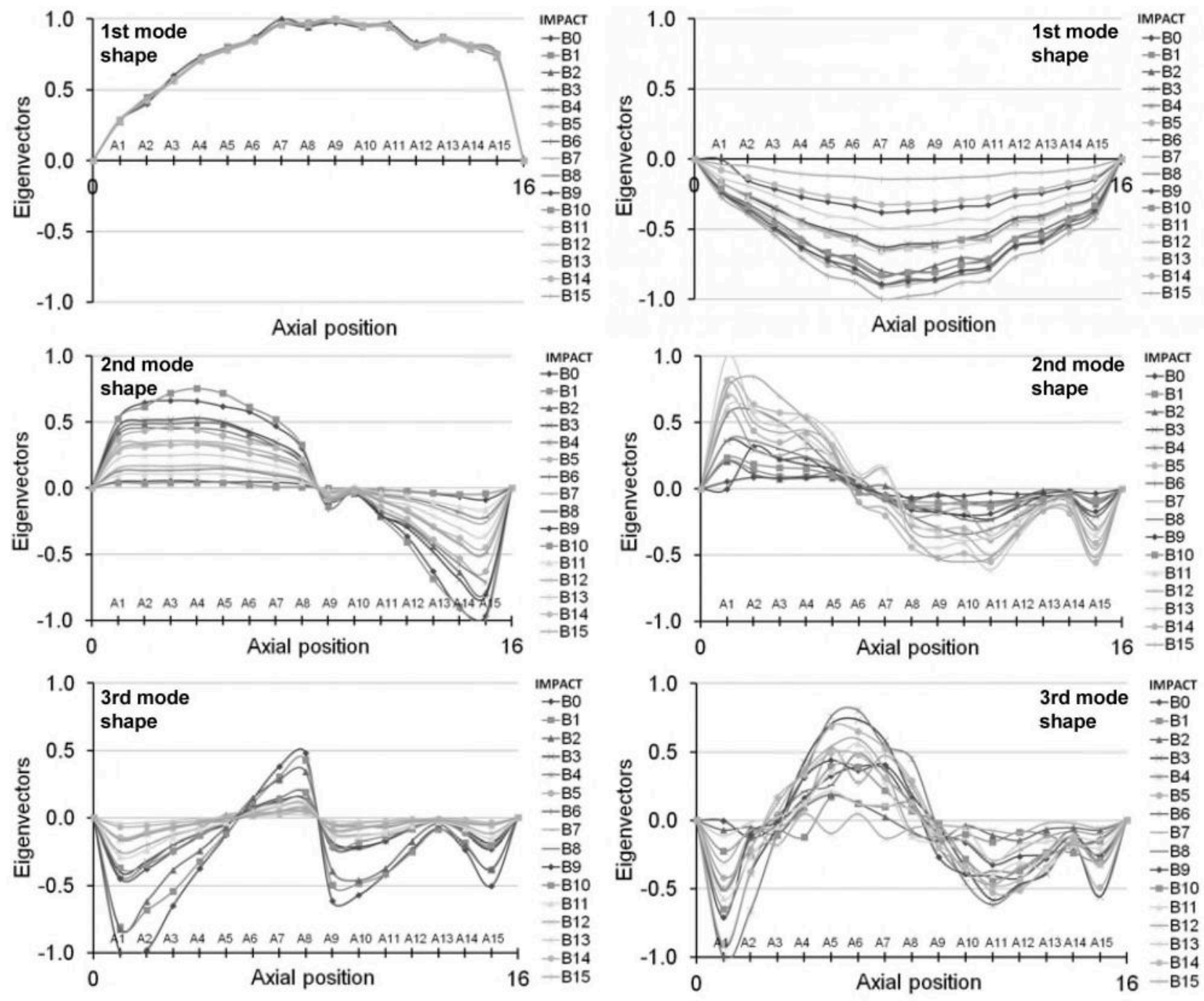

Along y axis, $\mathbf{J}_{\max }$

Along $\mathrm{x}$ axis, $\mathrm{J}_{\min }$ 
Figure 5. Experimental mode shapes

\subsection{Preliminar Numerical Model}

The anomalities detected by experimental modal analysis were investigated by preliminar numerical model.

An ANSYS® Mechanical APDL ${ }^{\mathrm{TM}}$ code was used for the numerical modal analysis. The FE (Finite Element) model has been built with 4800 8-noded, 6-DoF-per-node SHELL281 elements. The numerical model has been characterised by the physical and mechanical properties reported in Table 1. The reference system coincides with the one reported in Figure 2.

The structure was modelled considering 95 equally-spaced locations for the output channels, coincident with the nodes located as close as possible to the actual position of sensors (Figure 6).

Five FEMs were built, representing (i) the undamaged beam and (ii)-(v) the same structure with a triangular slot in the web at the point of connection with the lower flange, but not extended to it, with four depths - namely, $f=1,10,25$ and $50 \mathrm{~mm}$, out of a web total height of $180 \mathrm{~mm}$ (Figure 2). The base $c$ of the triangular shape was fixed equal to its height $(f=c)$; a notch was inserted at $z=3.65 \mathrm{~m}$ from the left end of the beam. Output channels were firstly considered along the $y$ direction then along $x$, to represent the two resting configurations of Figure 3. The modal analysis was then run on all FE models. Both mode shapes and numerically derived modal curvature were investigated.

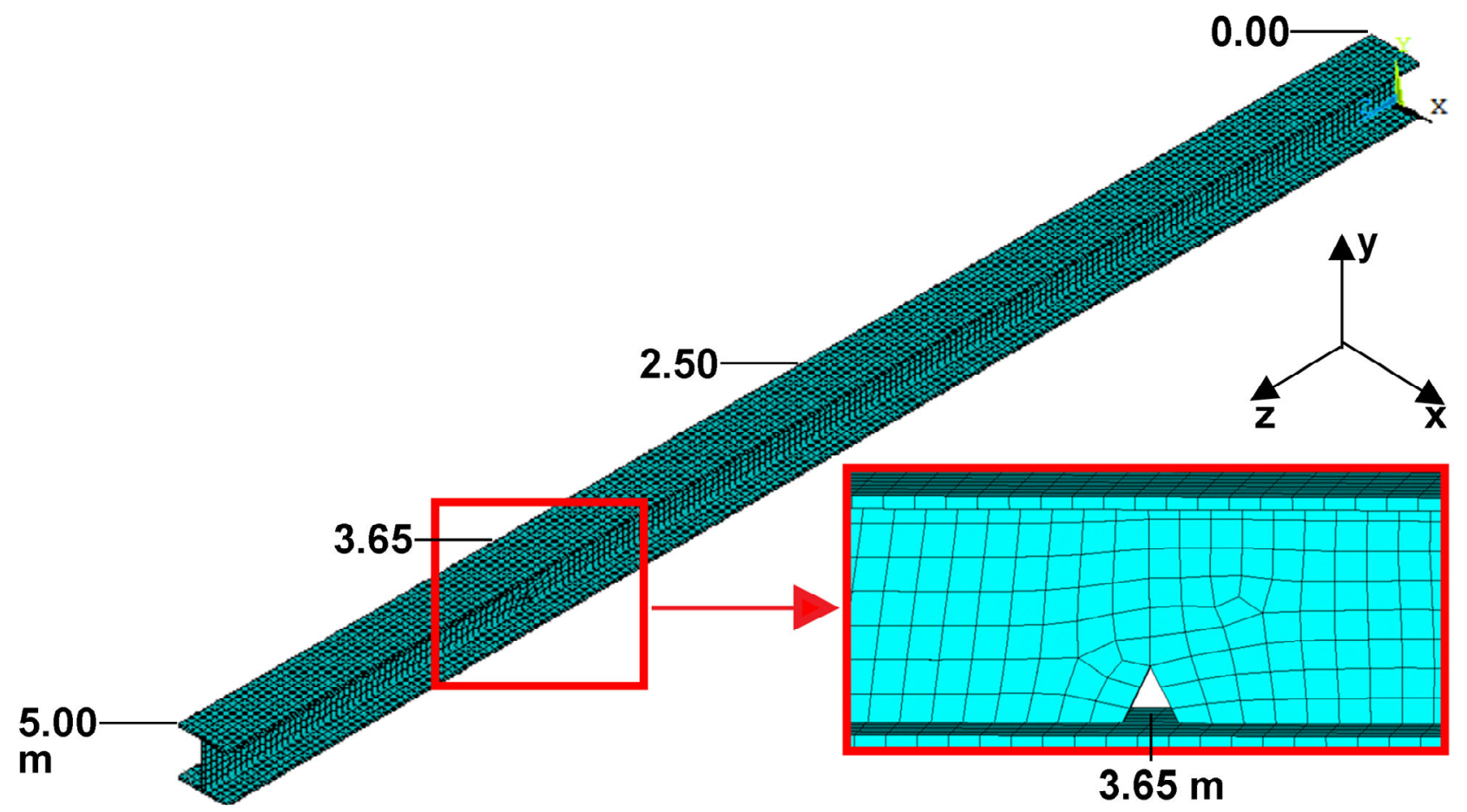


Figure 6. The triangular shape of the modelled slot. Four notch depths were considered: 1, 10, 25 and $50 \mathrm{~mm}$. The 50-mm-heigh notch is pictured here; note as it is not extended to the flange.

\section{APPLICATION OF TGP TO NUMERICAL AND EXPERIMENTAL MODE SHAPES}

\subsection{TGP for Numerical Mode Shapes}

Figure 7 shows the four mode shapes identified from the numerical model (for convenience only the model with the maximum notch size $50 \mathrm{~mm}$ is shown). For every damage level, four white Gaussian noise were taken into account with standard deviation equal to $\sigma_{n}=0.001,0.002,0.005$ and 0.01 .

All the 95 equally-spaced output channels were set as potential candidates for damage localisation. The statistic reliability of procedure has been assured by re-running the TGP routine 50 times for each combination of noise level and crack depth.

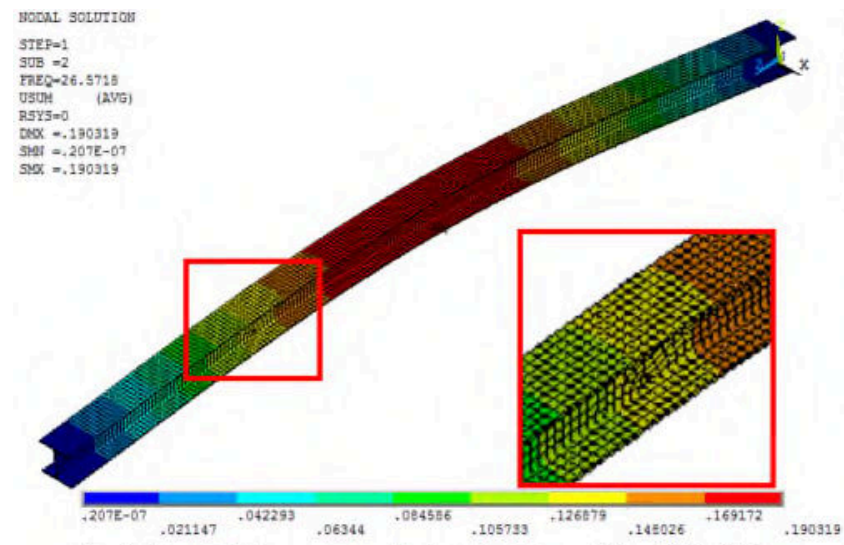

1st bending modal shape, $\mathrm{Hz} 26.57$

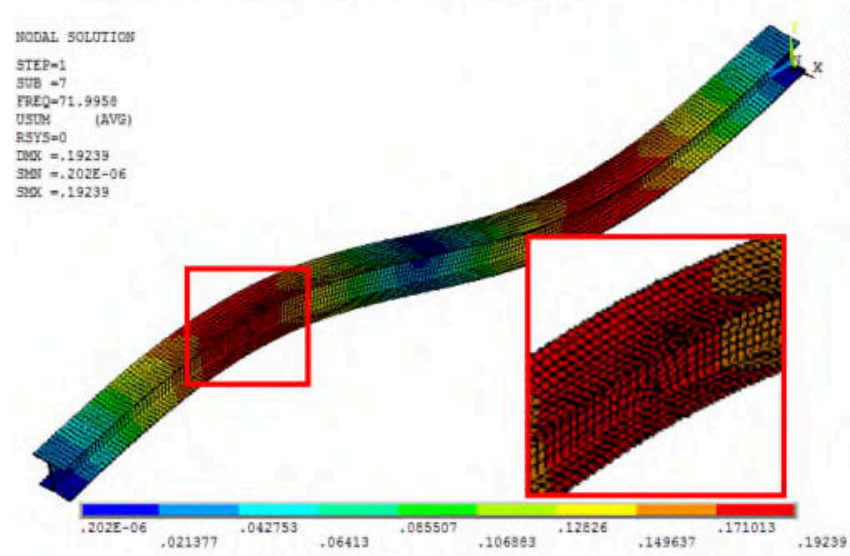

2nd bending modal shape, $\mathrm{Hz} 72.0$

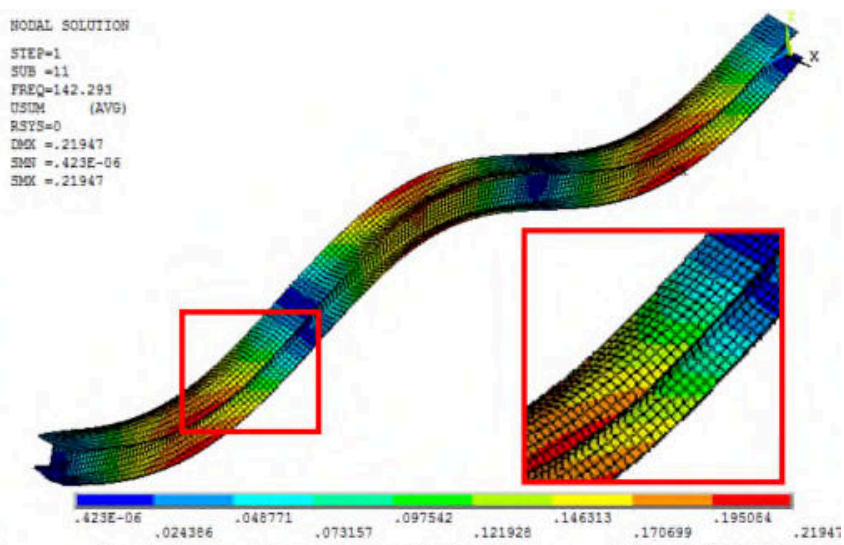

3rd bending modal shape, $\mathrm{Hz} 142.3$

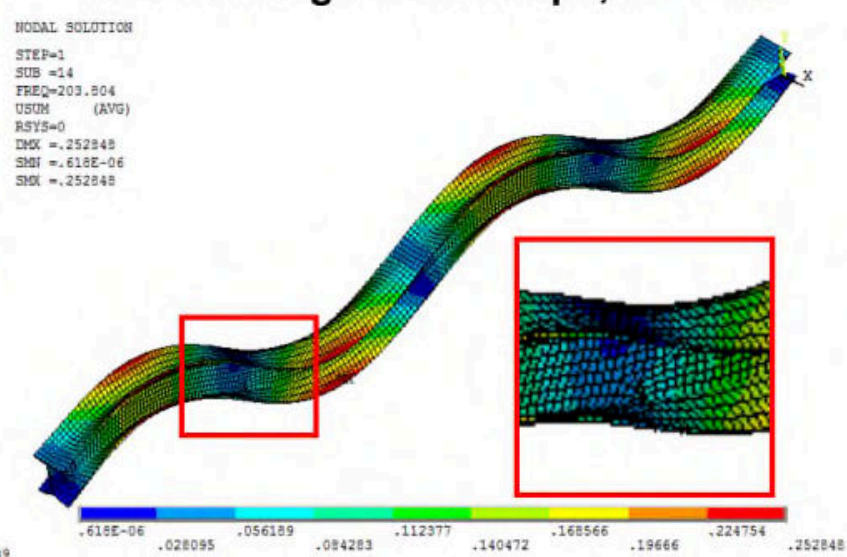

4th bending modal shape, $\mathrm{Hz} 203.8$

Figure 7. Damaged Mode Shapes (zoom on the damage reported in-box), maximum notch size (50mm). 
All numerical cases analysed are represented here by a selected case, the hammer test in position B7, for the sake of conciseness. Figures 8, 9 and 10 show the results of the first run (out of 50) for the output channels along the $J_{\max }$ direction. On the left of every figure, the input data (i.e., the raw mode shape or the modal curvature) is reported. For clarity, all bifurcations have been marked with the vertical lines which identify the consecutive GP regressions. As it can be seen, the algorithm is very sensible to even small variations of the input, thus returning many bifurcations, especially clustered around the antinodes of the mode shapes. This issue was dealt with by (1) focusing on the first branching only, which is the one most prominent, and (2) by focusing on the statistical results. This is shown on the right of Figures 8,9 and 10 by the power density function (pdf) of the first branching as obtained from the 50 reiterations of the procedure. The damage is identified by the dotted orange lines.

Figures 8 and 9 show, respectively, the consequences of noise on the raw data and their second derivatives (the second mode and the curvature of the fourth mode are depicted as an example, in this order). The smallest notch $(1 \mathrm{~mm})$ is considered. Figure 10 displays the effects on the curvature of changing the notch size; the comparison is run over the minimum level of noise $\left(\sigma_{n}=0.001\right)$ and for the third mode. The effects of increasing the damage size have been found to be negligible for raw mode shapes; the comparison is reported for completeness (Figure 11), for the highest level of noise in this case $\left(\sigma_{n}=0.01\right)$ and the first mode. 

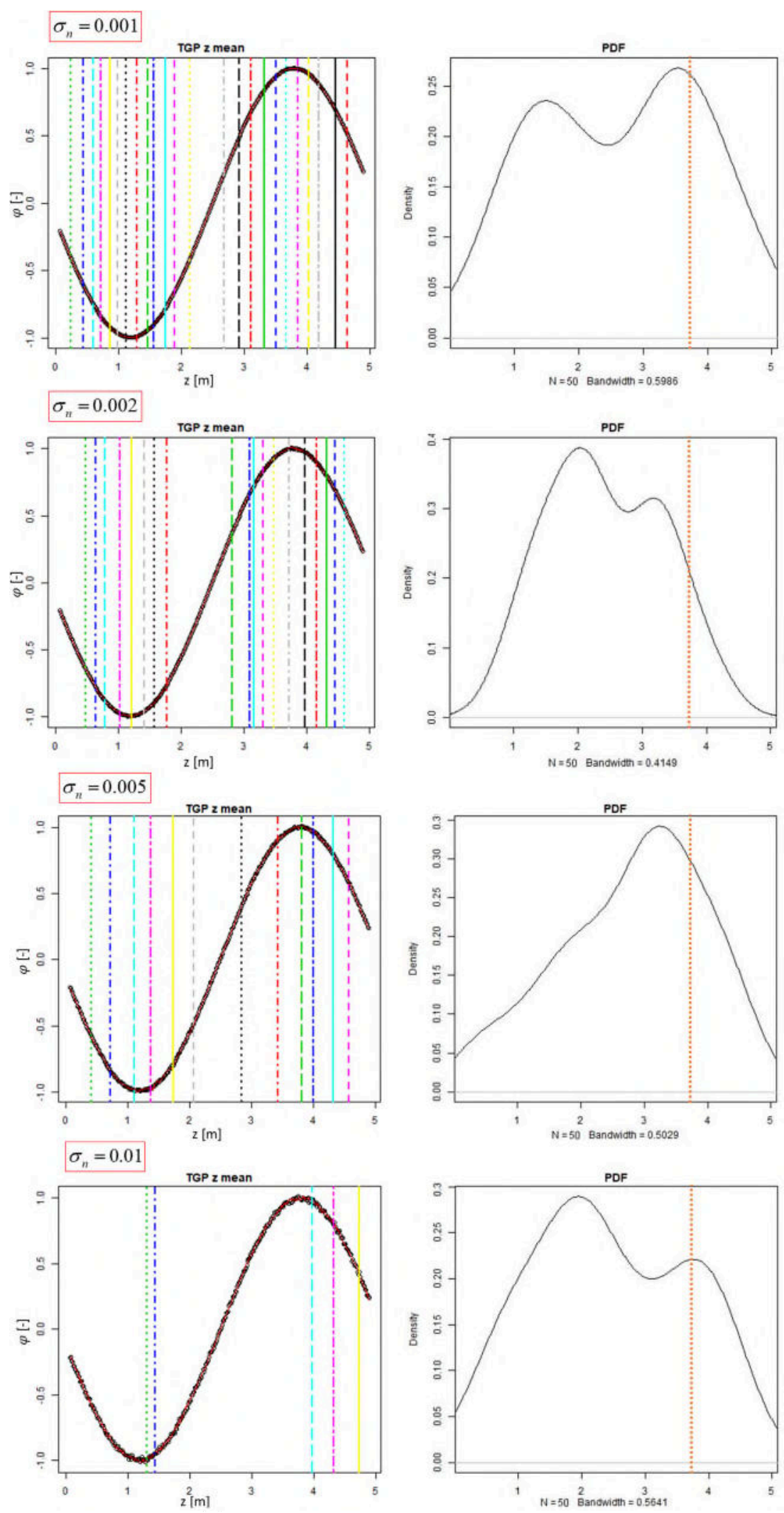

Figure 8. Notch $1 \mathrm{~mm}$, second mode shape. For $\sigma_{n}=0.001,0.002,0.005$, and 0.01. The dotted orange line indicates the actual damage location. 
The second mode shape amplifies the damage-related effects which are close to its second antinode. These effects are difficult to distinguish respect to the trend to bifurcate of TGP in the small noise condition; in correspondence of the first antinode, they are confused with the changes of the mode shape trend. These limits can be overcome by comparing more mode shapes as it will be explained later.

Further increasing the noise reduces the number of partitions, as the surrogate model becomes less overfitted. Nevertheless, the induced effects of a notch of minimal size remain too small to be efficiently discerned. These effects are improved when magnified via numerical differentiation (Figure 9). 

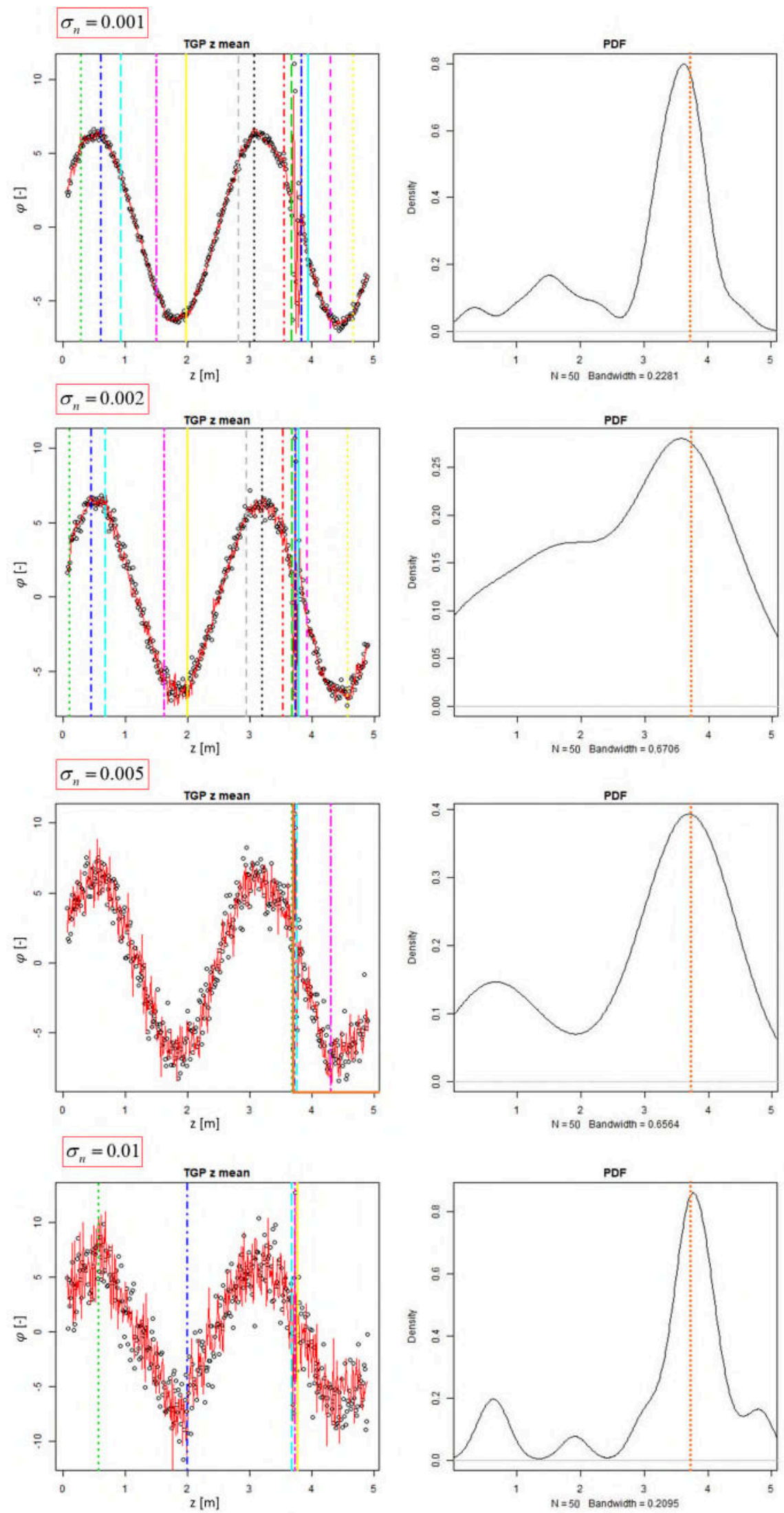

Figure 9. Notch $1 \mathrm{~mm}$, the curvature of the fourth mode shape. For $\sigma_{n}=0.001,0.002,0.005$, and 0.01. The dotted orange line indicates the actual damage location. 
By numerically deriving twice, both damage-related and noise effects are highly exalted. However, from the numerical example reported, it is noticeable how increasing the noise produced a nonmonotonic trend - damage becomes less visible by increasing $\sigma_{n}$ from 0.001 to 0.002 and then improve for the subsequent cases. Generally speaking, it was found that increasing the noise level causes the fourth mode (which has a more complex shape) to branch more times, while instead decrease the number of bifurcations for modes \#1, \#2 and \#3, which have overall 'simpler' eigenshapes. This can be due to a minimal optimum between less overfitting error on a more varied dataset and more elaborate function to represent. 

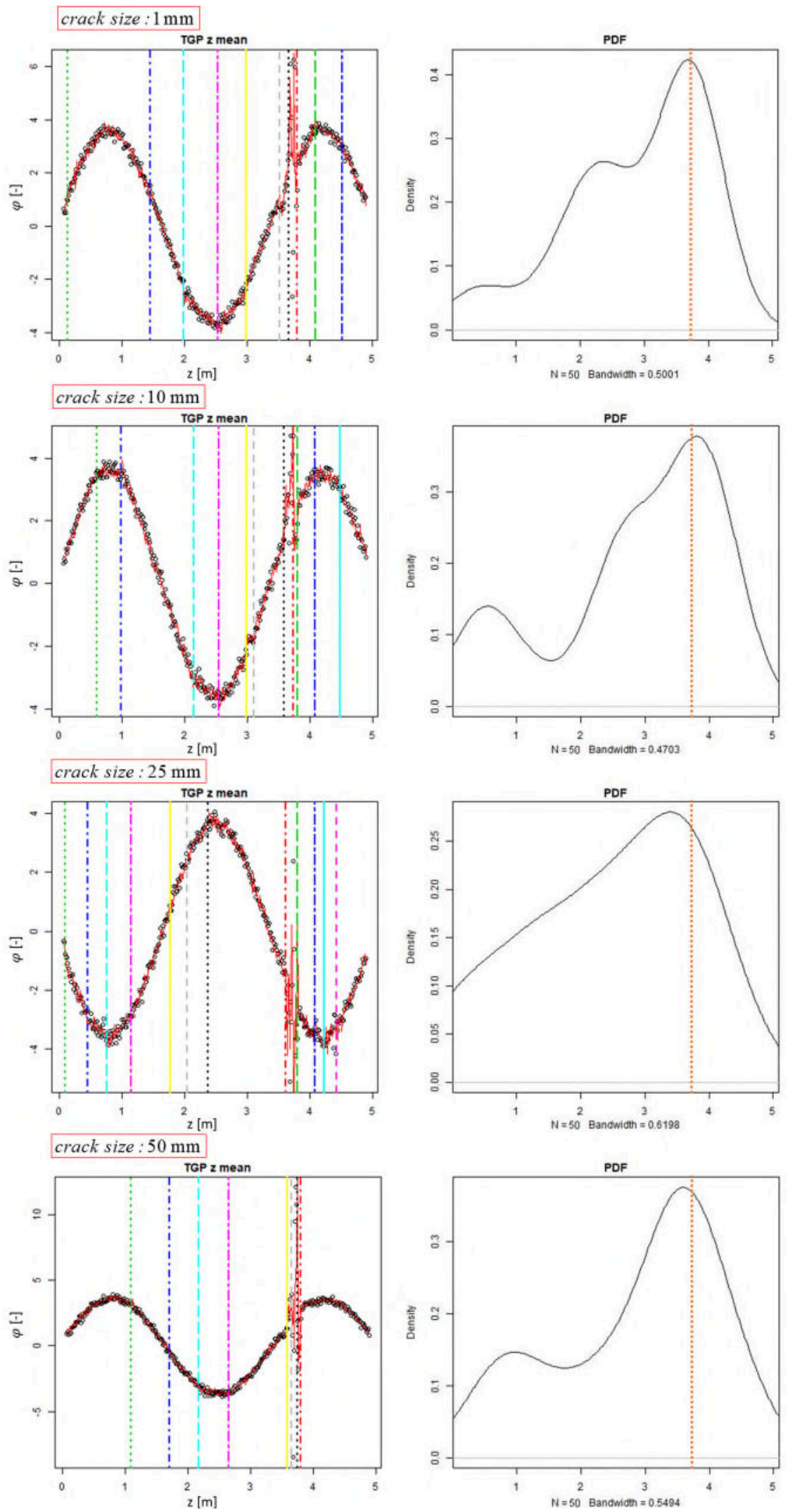

Figure 10, noise equal to $\sigma_{n}=0.001$, the curvature of the third mode shape. For crack sizes 1,10 ,

25 , and $50 \mathrm{~mm}$. The dotted orange line indicates the actual damage location. 
The effects of notch size are much less pronounced than the ones induced by artificially-added noise, as can be inferred from Figure 10. Regarding the curvature of the third mode, passing from 1 to $10 \mathrm{~mm}$, the difference in the first branch distribution is not very evident. The notch position becomes clearer when the size is further enlarged. Otherwise, the notch is hardly visible in the first mode shape (Figure 11), independently from its depth; partitions of the first mode are overwhelmingly located at the midlength, due to the global maximum of the mode shape at L/2. 

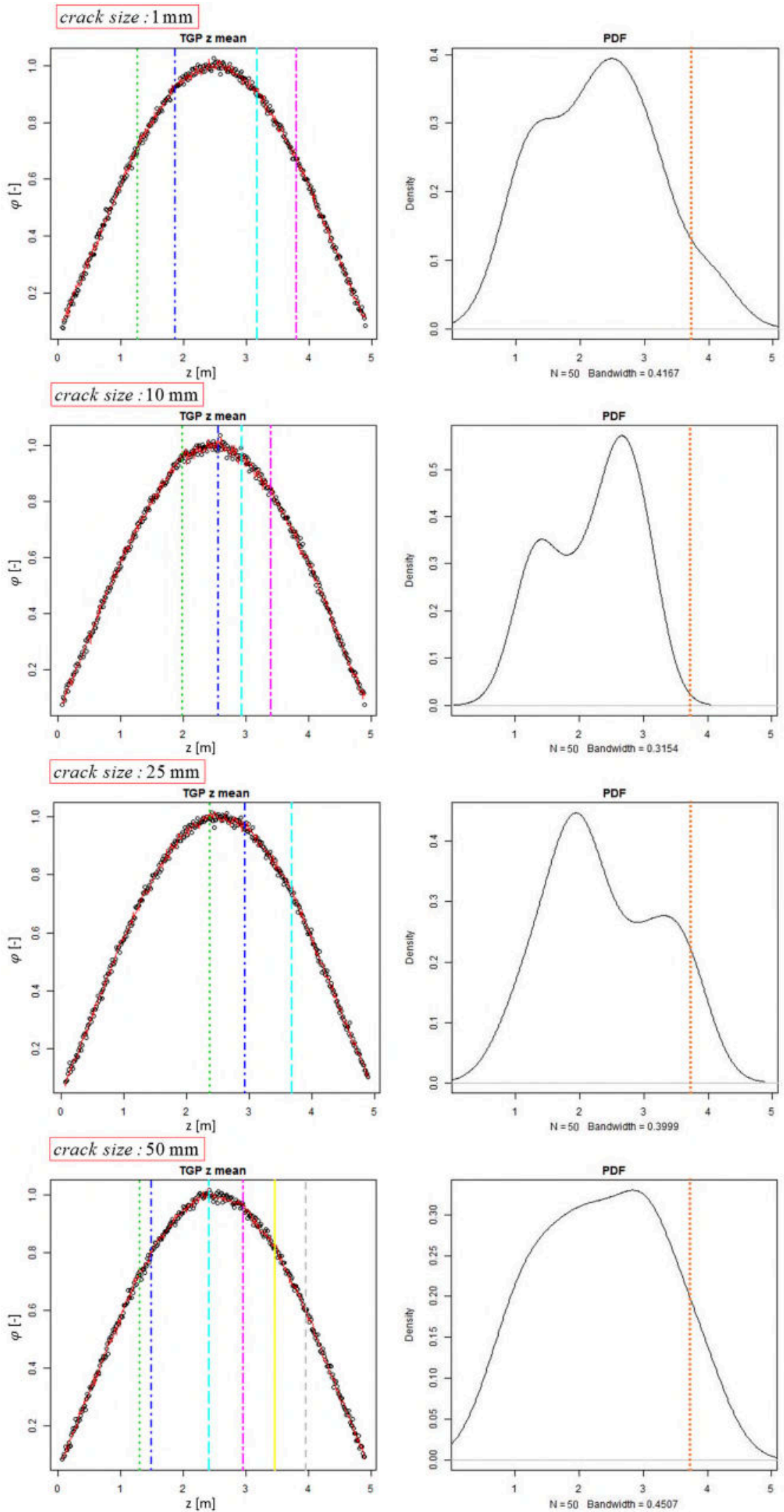

Figure 11 , noise equal to $\sigma_{n}=0.01$, first mode shape. For crack sizes $1,10,25$, and $50 \mathrm{~mm}$. The dotted orange line indicates the actual damage location. 
The numerical modal data highlight the capacity of a small amount of independent and identically distributed noise to stabilise the branching fitting on data. It's possible to identify a denser area than a unique partition corresponding to the imperfection location.

Very importantly, it can be noticed how erroneous branching tend to cluster around mode shapes' antinodes (i.e., the local maxima and minima). In particular, it is evident that when the first branching point is not related to the notch presence, it is related to an antinode. Often, the antinode closer to the beam mid-length is the one to be firstly localised, which becomes particularly evident for the first eigenshape, for both larger and smaller notches.

To deal with the issue, evident blunders had to be removed from the results before carrying any further statistical study on them. The post-processing strategy adopted was to compare any mode shape with the other three for the same input. The algorithm simply discards all the branching that are both (i) included only in one mode shape and not in the others and (ii) are close to one of that mode shape's extrema. In this way, erroneous misallocations at the antinodes of the mode shapes were greatly reduced. Comparison between different mode shapes from the same damaged structure is frequently used for damage localisation, as done as, for instance, in [41]. All the so-obtained results are schematized in Figure 12.

Considering the different notch sizes for the for mode shapes of both directions, the average values and the standard deviations for every noise level $\sigma_{\mathrm{n}}$ have been represented respectively by the dots and lines in Figure 12; the length of the lines represents one standard deviation of the pdf.

As it can be seen there, the mean value of the first branching was found to be not coincident for mode shapes extracted along the $\mathrm{x}$ - and the $\mathrm{y}$-direction. This was found for all the damage conditions and all the four mode shapes. Results along the y-axis generally returned more precise results. The best result was achieved for the case with the $50 \mathrm{~mm}$ notch, i.e. for the maximum depth considered for the damage, as easily predictable. These results show minimal difference between the average value and the actual damage location and low standard deviation. 

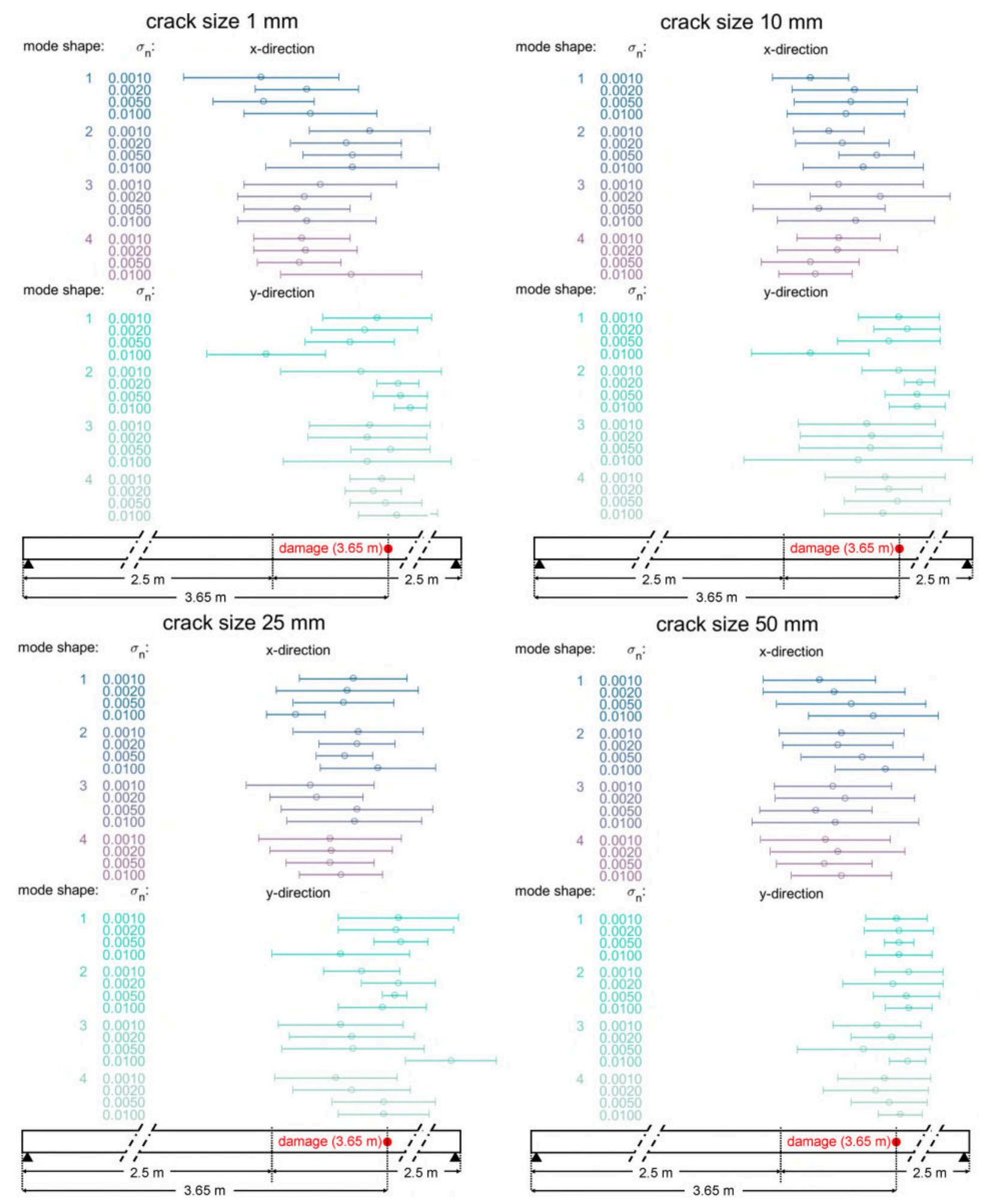

Figure 12. Statistical results of the numerical simulations

\subsection{TGP for Experimental Mode Shapes}

From preliminary results, it was found that using the modal curvatures derived twice from the experimental mode shapes did not produce any improvement in the results. This is most probably 
due to the higher level of noise and the low number of recording channels. Thus, only the experimental mode shapes are here reported.

The first four mode shapes were analysed, computed along $x$ - and $y$-directions, for sixteen hammer blows and roving the point of application; 128 cases were therefore studied. The comparison between the several hammer blows is crucial, as small, unintentional variations in strength and orientation when landing may produce uncertainties in the recorded measurements. Some of the data recorded were very poor, due to clipping. In this case, the signals were properly truncated and the part of signals saturated was discarded. Thus, it was not possible to identify correctly mode shape \#2 with hammer blows at point \#6 along $x$ - and $y$-direction, and at points \#8, \#9, \#11 and \#13 along $x$-direction; mode shape \#3 with input applied at point \#7 along $x$-direction and at points \#3, \#5, \#10, \#11, \#12 and \#16 along y-direction; and finally, mode shape \#4 at points \#3, \#9, \#10, \#15 and \#16 (only along $x$-direction).

Due to high measurement noise, some cases presented very deep trees, with many bifurcations. To overcome this issue, it was decided to take into account only the first branch, as also previously done for the numerical simulations.

To validate statistically the results, each case was re-run 100 times. Results in term of mean and standard deviation of the first branch of each case are schematised in Figure 13. The TGP proved to often incorrectly detect maxima and minima as switching points, as for the numerical case; thus, the same strategy previously described - that is to say, benchmarking the mode shapes against one another to remove branching points at the antinodes - was adopted. 
mode shape 1

hammer strike number: $\quad x$-direction

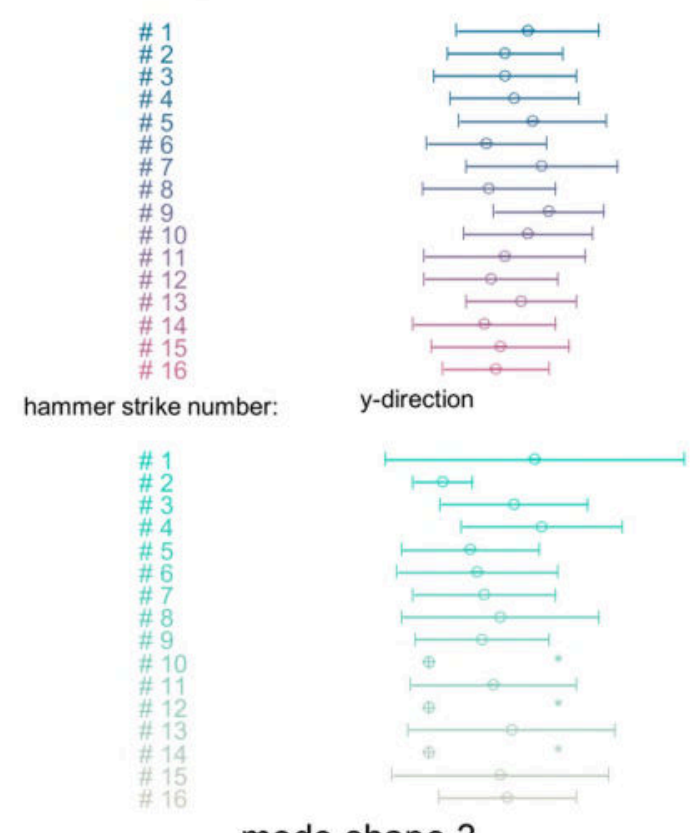

mode shape 3

hammer strike number: $\quad \mathrm{x}$-direction

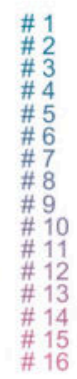

hammer strike number: $\quad y$-direction
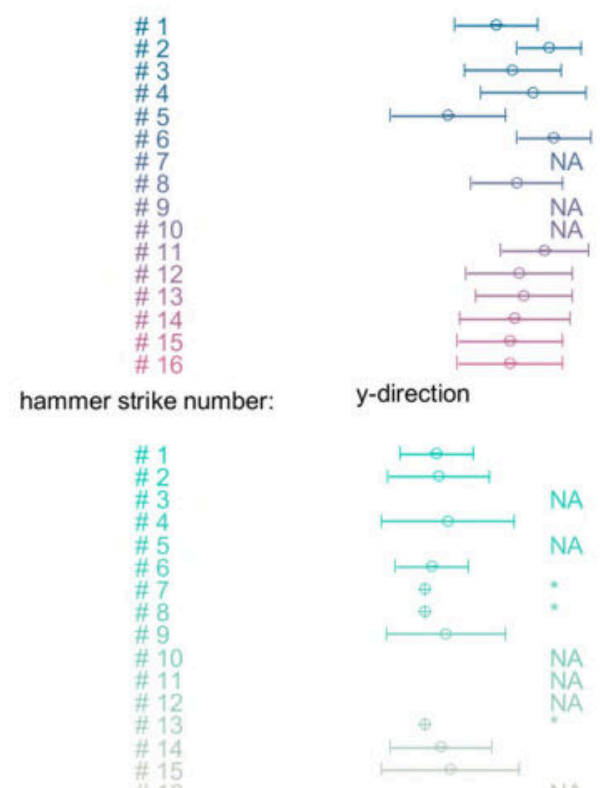

mode shape 2

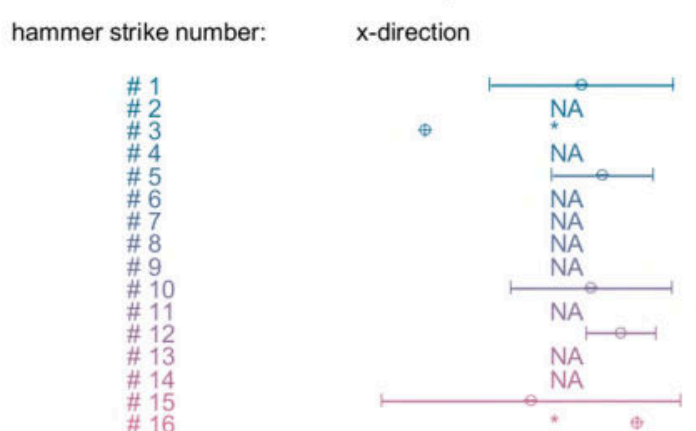

hammer strike number:

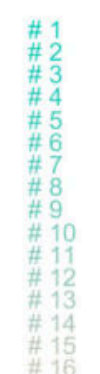

mode shape 4

hammer strike number: $\quad$-direction

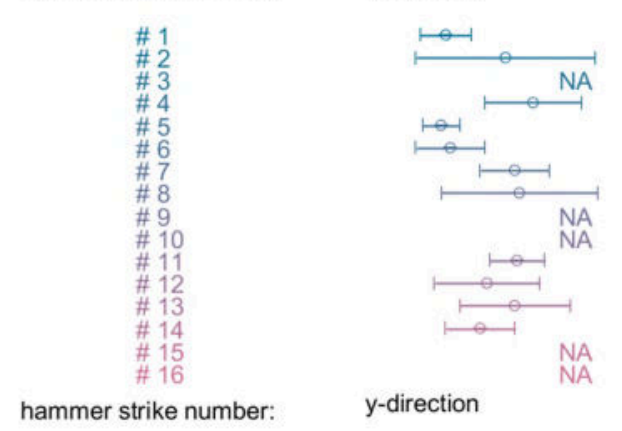

hammer strike number:

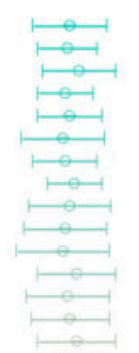

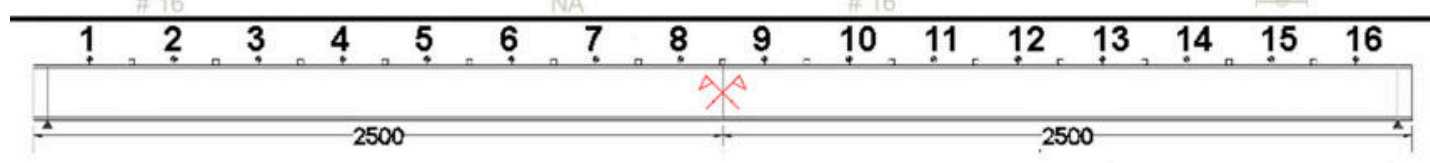

Figure 13. Statistical results from the experimental data: mean values and standard deviation. The symbol (*) shows the presence of only one result and the consequent null variance, while NA labels the not available data. 
All hammer blows produced quite consistent results for all mode shapes, with a mean value ranging from a minimum of $\mathrm{z}=2.80 \mathrm{~m}$ and a maximum of $\mathrm{z}=3.49 \mathrm{~m}$. This seems to indicate a major region of disturbance in this $\sim 0.7 \mathrm{~m}$-long tracts of the beam. The mode shapes 1 and 3 in the $\mathrm{x}-$ direction and the mode shapes 2 and 4 in y-direction show the most similar results.

The overall average indicates a location (for the first branch) around $3.16 \mathrm{~m}$, with a standard deviation of $0.21 \mathrm{~m}$. The third Mode shapes with hammer blown along the y-direction produced some estimations generally slightly skewed to lower values respect to the other means; vice versa, the fourth mode shapes (again hammered in the y-direction) gave slightly larger results. Not considering the results from both these two sets, the average becomes $3.15 \mathrm{~m}$ with a slight reduction of the standard deviation $(0.18 \mathrm{~m})$; hence, circa $99 \%$ of the values (assuming a Gaussian distribution and considering, therefore, a $3 \sigma$ interval of confidence) falls in between the $\mathrm{z}=2.62 \mathrm{~m}$ $3.68 \mathrm{~m}$ range. It is reasonable to say that the empirical data localise some discrepancies in this portion of the beam length.

It must be noted that results may be partially misled by the prominence of good identification and good partitioning for the first (both along $x$ - and $y$-directions) and for fourth (along $y$-direction) eigenshapes. The second and the third mode were more affected by insufficient identification and unbranched trees, thus they are less represented in the final values of mean and standard deviation, computed by averaging all the results.

Some results from hammer blow \#1 in the $x$-direction and hammer blow \#1 in the $y$-direction are here reported in Figures 14 and 15, respectively, and commented as an example; all discussions can be extended to similar cases. The portraited regressions and decision trees are used only to visualise the TGP output and are not to be intended as representative of the most common ramifications for their respective modes.

Figure 14a and Figure 14b both present erroneous branches at extrema of the mode shape, due to function inverting is trending. These were removed in post-processing. Conversely, Figure 14c and Figure $14 \mathrm{~d}$ are representative of what would be considered excellent runs, even if the fitting is not always locally accurate, as can be observed in Figure 14d in the second GP Regression (on the right side), which is not very satisfactory. Note that in Figure 14a the switching point considered to be imperfection-related is the first to branch; this was often observed in most of the cases, but not everywhere: in Figure 14b, the expected output is the last branching point, preceded by both the minimum and the maximum, in this order. 


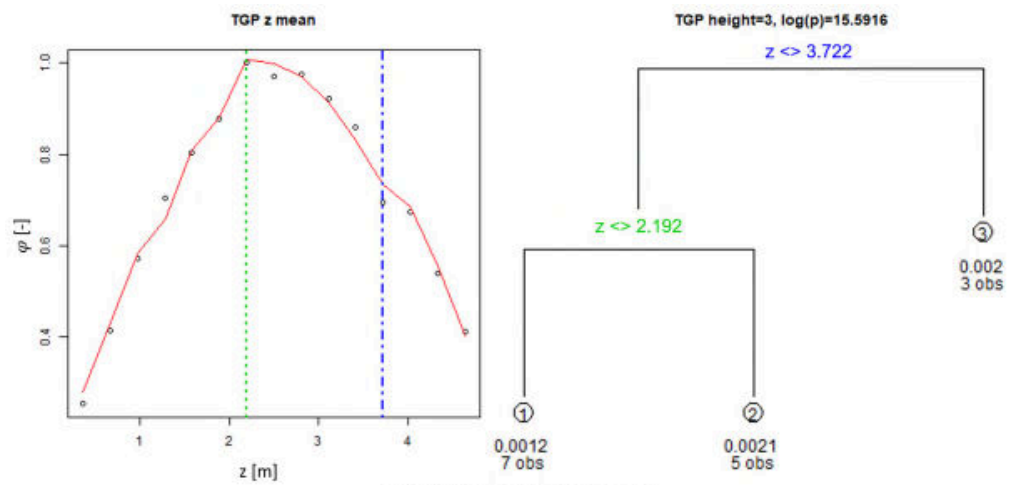

(a) Mode $1 \mathrm{x}$-direction

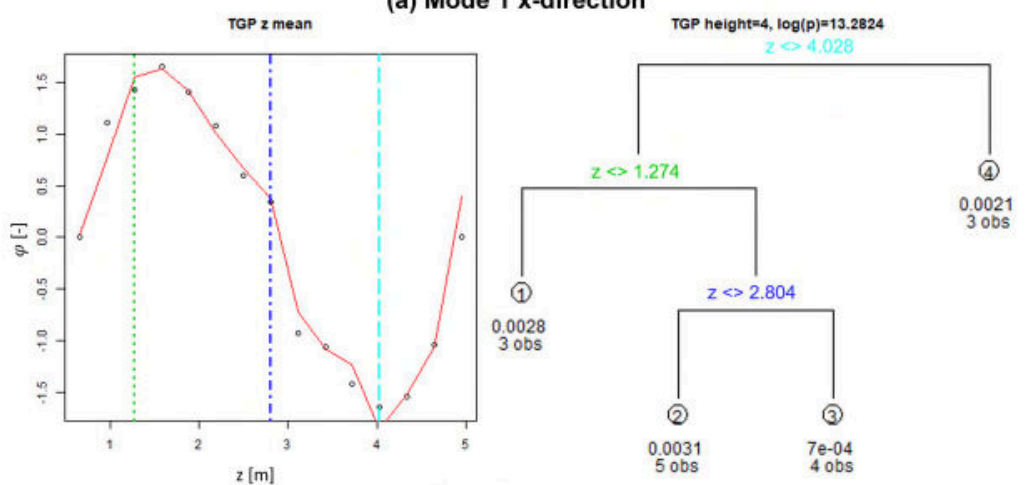

(b) Mode 2 x-direction

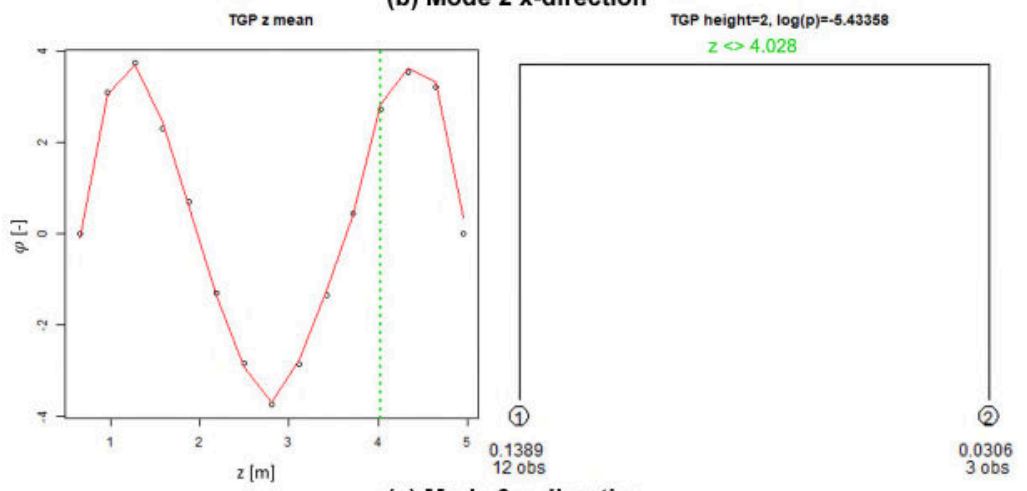

(c) Mode 3 x-direction

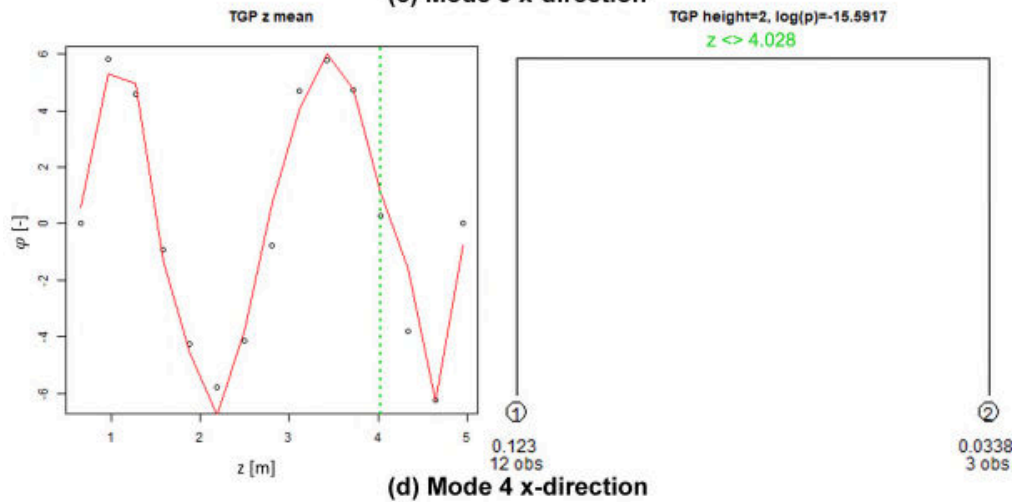

Figure 14. TGP regressions (solid red lines) over experimental data (black circles) collected by hammering in the $\mathrm{x}$-direction (on the left); dashed lines highlight the branching points at the boundary between subsequent GPs. Right: the corresponding hierarchical dendrogram of GP fittings. Branching points are coloured accordingly to their corresponding dashed lines. 
In Figure 15a, the expected imperfection-related branching comes after an erroneous partition; Figures $15 \mathrm{~b}$ to $15 \mathrm{~d}$ show an example of an accurate 1-partition tree on mode $\# 2$, and two inaccurate fittings on modes \#3 and \#4 along the y-direction.
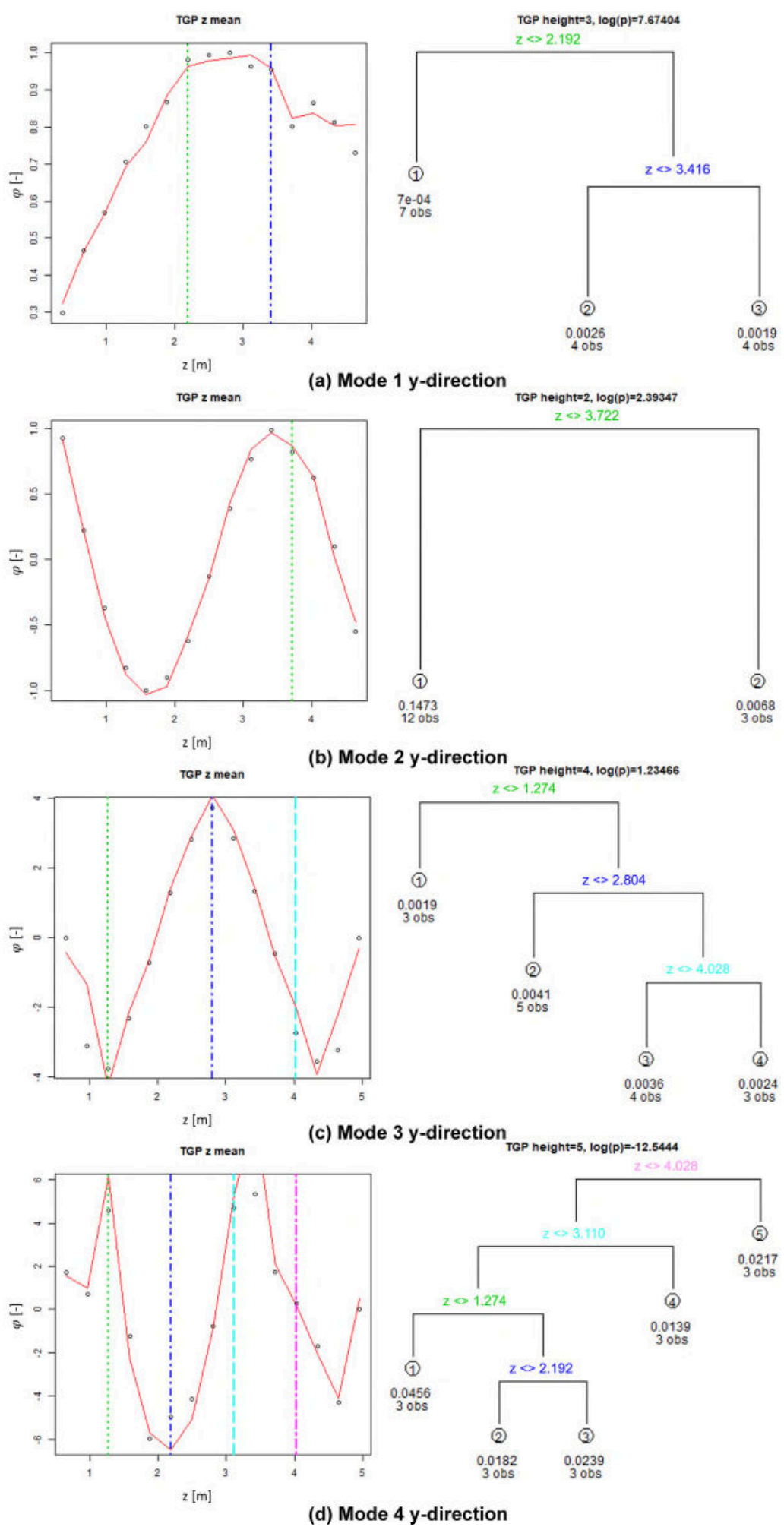
Figure 15. Left: TGP regressions (solid red lines) over experimental data (black circles) collected by hammering in the y-direction. Right: the corresponding hierarchical dendrogram of GP fittings. Branching points are coloured accordingly to their corresponding dashed lines.

\subsection{Benchmarking against buckling testing}

The buckling tests carried out on the specimens obtained by the profile with wide flanges analysed in this paper allow to verify the reliability of procedure proposed in this work. Both specimens, $\mathrm{H} 1$ and H2, long 2.5 meters (Figure 16), of the whole pultruded profile long 5 meters, were tested to evaluate the buckling phenomena through hinge-hinge configuration, Figure 17a.

As shown in Figure 17b the sample $\mathrm{H} 1$ is affected by global buckling behaviour while in the $\mathrm{H} 2$ profile the local buckling has been triggered by the degradation of web-flange interaction (Figure 17c). This latter phenomenon is characteristic of thin-walled profile with orthotropic constitutive configuration. In detail, in the $\mathrm{H} 2$ profile the intrinsic imperfection at $3650 \mathrm{~mm}$, identified by TGP, triggers the local failure mechanism as shown in Figure 17d.

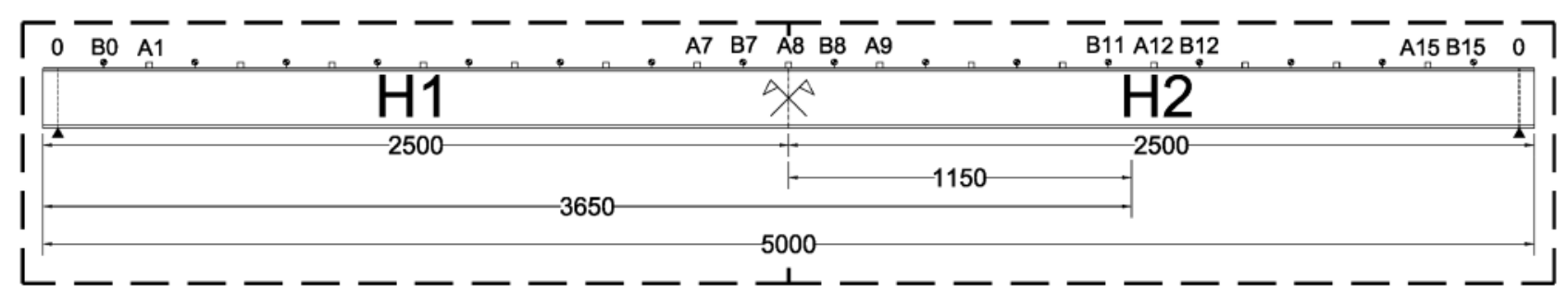

Figure 16, $\mathrm{H} 1$ and $\mathrm{H} 2$ samples of wide flange profile (see Figure 5) 


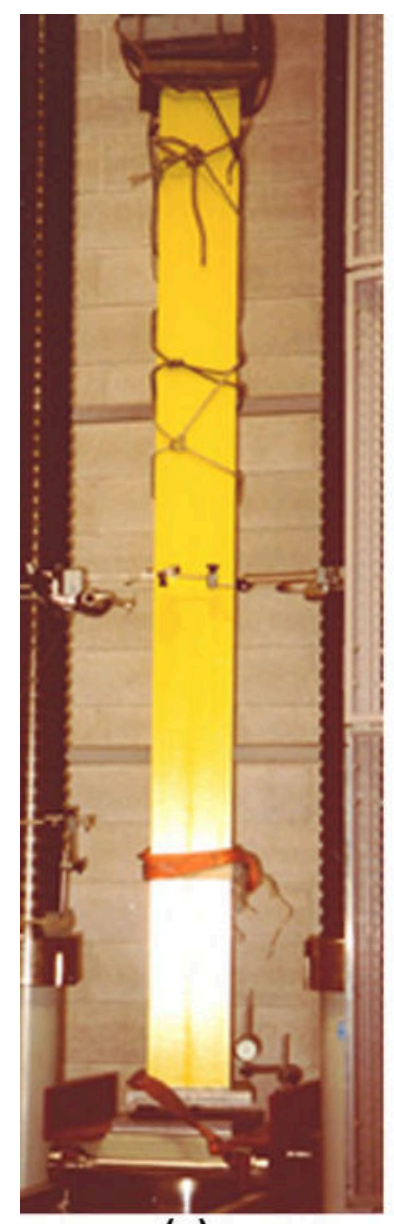

(a)
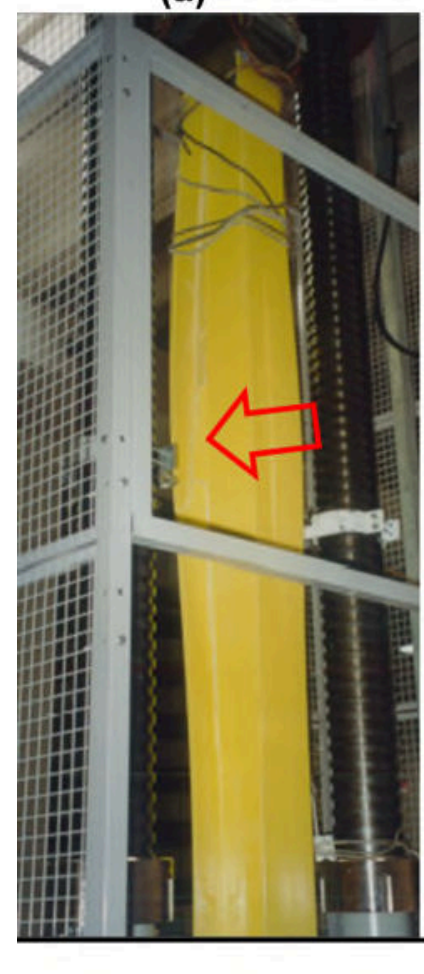

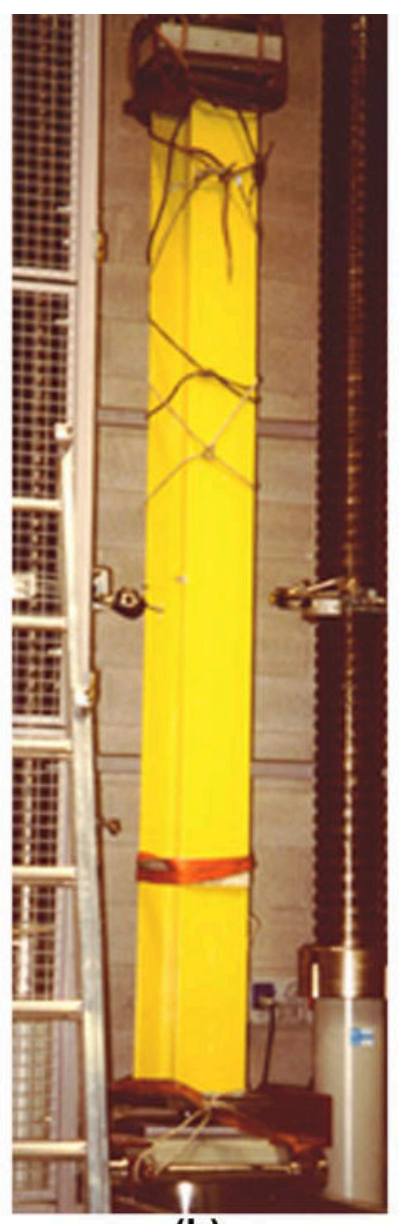

(b)

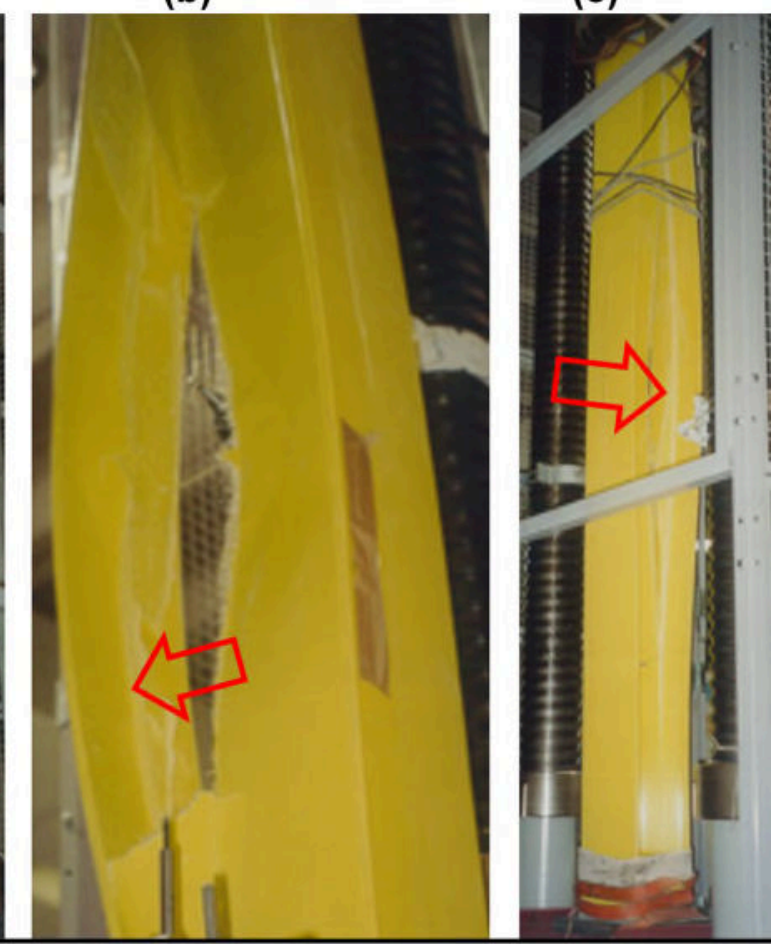

(d)

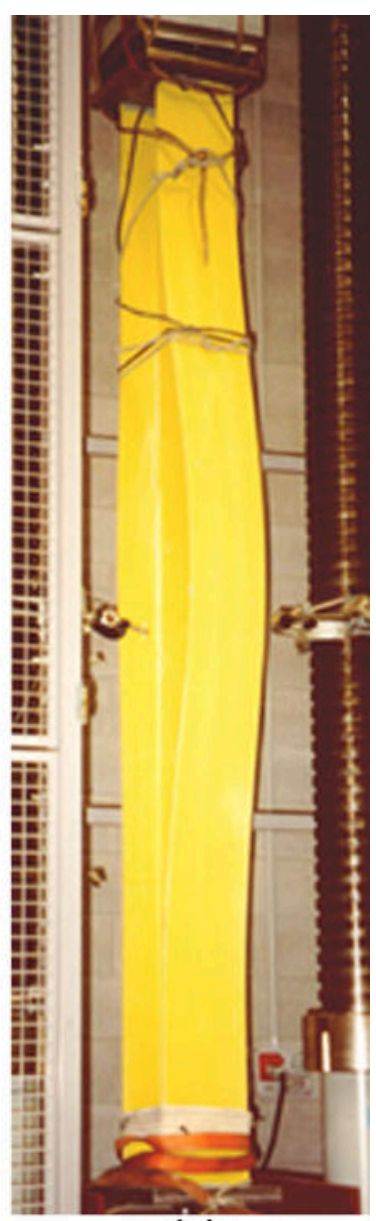

(c)
Figure 17, (a) buckling test setup, (b) global buckling of H1 sample, (c) local buckling of H2 sample, (d) local failure mechanism of $\mathrm{H} 2$ sample 


\section{CONCLUSIONS}

This research included two case studies: an experimental case of a pultruded GFRP beam with manufacturing imperfections and a FE model of the same beam with a notch. Both were investigated to assess the capabilities of Treed Gaussian Processes (TGPs) for the detection and localisation of discontinuities in the mode shapes, which can in turn be linked to the presence of damage or material defects. In the experimental case study, no damage was visible by eye or by other means of direct inspection; yet, the experimental modal analysis highlights the anomalies in mode shapes that are generally related to intrinsic imperfections. This was further corroborated by the results of the buckling tests.

The potential of the TGP procedure has been evaluated numerically and experimentally, in the latter case for both the main axis of thin-walled GFRP pultruded profile. The following conclusions can be drawn:

- the discontinuities detected in all the four first mode shapes both along $\mathrm{x}$ - and $\mathrm{y}$-axes identify a corrupted part of the beam. This portion can be quite certainly affected by imperfections;

- This area corresponded to the tract where the buckling initiated during the buckling test of the test beam;

- the first two mode shapes demonstrate the effectiveness of the proposed method with a satisfactory level of accuracy;

- for the highest mode shapes the discontinuities identification is difficult due to the their complexity; also, the higher mode shapes are partially affected by torsional phenomena and are consequently out-of-plane mode shapes, more sensible to the orthotropic behaviour of pultruded thin-walled profile;

- restricting the study to the first branch (which is the most discriminative) enables to improve the procedure. Nevertheless, the experimental and numerical results show the sensitivity of the model to overfitting producing branch at antinodes; this aspect is affected by the inability of shape function to distinguish local discontinuities and global trends.

- the comparison of two or more mode shapes for the same input solves this uneasiness. The presence of partitions in only one of two or more mode shapes enables to discard it;

- All imperfection/damage identification techniques are sensitive to measurement noise such as the proposed TGP method; 
- The bad effects of a high level of noise have been demonstrated by the numerical results. The analysis of data without noise are more reliable than highly noisy ones; even if an adequate noise level enables TGP to avoid both overfitting and erroneous branching.

\section{Acknowledgements}

The authors wish to thank Mr Pier Paolo Cosentino for his support during the processing data phase.

\section{References}

1. Barbero EJ. Introduction to Composite Materials Design. Taylor and Francis, Philadelphia, PA, 1st edition, 1999.

2. Anonymous. Strongwell Design Manual. Strongwell Composites Inc, Bristol, VA. (www.strongwell.com);

3. Anonymous. The Pultrex Pultrusion Design Manual (Imperial version) Vol. 4 Rev. 6. Creative Pultrusion Inc., Alum Bank, PA. (www.creativepultrusions.com/library.html)

4. Nguyen TT, Chan TM, Mottram JT. Influence of boundary conditions and geometric imperfections on lateral-torsional buckling resistance of a pultruded FRP I-beam by FEA. Composite Structures 2013; 100:233-242.

5. Donnell LH, Wan CC. Effect of Imperfections on Buckling of Thin Cylinders and Columns Under Axial Compression. Journal of Applied Mechanics 1950;17(1):73-83.

6. Cohen GA, Haftka RT. Sensitivity of Buckling Loads of Anisotropic Shells of Revolution to Geometric Imperfections and Design Changes. Computers \& Structures 1989; 31(6):985-995.

7. Starnes JH, Jr., Hilburger MW, Nemeth MP. The Effects of Initial Imperfections on the Buckling of Composite Cylindrical Shells. In STP1383-EB Composite Structures: Theory and Practice, edited by P. Grant and C. Rousseau, (pp. 529-550). West Conshohocken, PA: ASTM International, 2001. doi:10.1520/STP14529S.

8. Wang B, Ma X, Hao P, Sun Y, Tian K, Li G, Zhang K, Jiang L, Guo J. Improved knockdown factors for composite cylindrical shells with delamination and geometric imperfections. Composites Part B: Engineering 2019; 163:314-323.

9. Kepple J, Herath MT, Pearce G, Prusty BG, Thomson R, Degenhardt R. Stochastic analysis of imperfection sensitive unstiffened composite cylinders using realistic imperfection models. Composite Structures 2015;126:159-173.

10. Boscato G, Ientile S. Experimental and numerical investigation on dynamic properties of thin-walled GFRP buckled columns. Composite Structures 2018; 189(1):273-285. doi.org/10.1016/j.compstruct.2018.01.061.

11. Farrar CR, Worden K. Structural Health Monitoring: A Machine Learning Perspective. Wiley, New York, 2013.

12. Doebling SW, Farrar CR, Prime MB, Shevitz DW. Damage Identification and Health Monitoring of Structural and Mechanical Systems from Changes in their Vibration Characteristics: A Literature Review. Los Alamos National Laboratory report, 1996 (LA-13070-MS).

13. Sohn H, Farrar C, Hunter N, Worden K. A Review of Structural Health Monitoring Literature: 1996-2001. Los Alamos National Laboratory report, 2003 (LA-13976-MS).

14. Carden EP, Fanning P. Vibration based condition monitoring: A review. Structural Health Monitoring 2004, 3, $355-377$.

15. Civera M, Zanotti Fragonara L, Surace C. A novel approach to damage localisation based on bispectral analysis and neural network. Smart Structures and Systems. Volume 20, Issue 6, December 2017, Pages 669-682.

16. Morassi A, Rollo M. Identification of two cracks in a simply supported beam from minimal frequency measurements. Journal of Vibration and Control 2001, 7(5), 729-739. 
17. Martucci D, Civera M, Surace C, Worden K. Novelty detection in a cantilever beam using extreme function theory. In J. Phys. Conf. Ser 2018 (Vol. 1106, No. 1, pp. 12-27).

18. Civera M, Zanotti Fragonara L, Surace C. An experimental study of the feasibility of phase-based video magnification for damage detection and localisation in operational deflection shapes. Strain 2020, 56(1), e12336.

19. Worden K, Cross EJ. On switching response surface models, with applications to the structural health monitoring of bridges. Mechanical Systems and Signal Processing 2018; 98:139-156. https://doi.org/10.1016/j.ymssp.2017.04.022

20. Worden K, Cross EJ, Brownjohn JMW. Switching Response Surface Models for Structural Health Monitoring of Bridges. In Koziel S., Leifsson L. (eds) Surrogate-Based Modeling and Optimization, Springer, New York, NY, 2013.

21. Zhang T, Barthorpe R, Worden K. A simplified treed gaussian process approach to the modelling of bridge data for structural health monitoring. Structural Health Monitoring 2017. https://doi.org/10.12783/shm2017/13910.

22. Civera M, Surace C, Worden K. Detection of Cracks in Beams Using Treed Gaussian Processes. In Niezrecki C. (eds) Structural Health Monitoring \& Damage Detection, Volume 7. Conference Proceedings of the Society for Experimental Mechanics Series. Springer, Cham, 2017.

23. Rasmussen CE, Williams C. Gaussian Processes for Machine Learning. The MIT Press, New York, 2006.

24. Hensman, J., Mills, R., Pierce, S. G., Worden, K., \& Eaton, M. (2010). Locating acoustic emission sources in complex structures using Gaussian processes. Mechanical Systems and Signal Processing, 24(1), 211-223.

25. Hensman JJ, Surace C, Gherlone M. Detecting mode-shape discontinuities without differentiating - examining a Gaussian process approach. In Proceedings of 9th International Conference in Damage Assessment (DAMAS 2011), Oxford (2011).

26. Corrado N, Durrande N, Gherlone M, Hensman J, Mattone M, Surace C. Single and multiple crack localization in beamlike structures using a Gaussian process regression approach. In Journal of Vibration and Control 2019; 24(18):4160-4175.

27. Breiman, L., Friedman, J., Stone, C.J., Olshen, R.A.: Classification and Regression Trees. Chapman and Hall/CRC, Boca Raton (1984)

28. Chipman HA, George EI, McCulloch RE. Bayesian cart model search. Journal of the American Statistical Association 1998; 93(443):935-948.

29. Chipman, H.A., George, E.I., McCulloch, R.E.: Bayesian treed models. Mach. Learn. 48, 299-320 (2002)

30. Gramacy RB. Bayesian treed Gaussian process models (Doctoral dissertation, University of California, Santa Cruz), Chap $5,2005$.

31. Becker WE. Uncertainty propagation through large nonlinear models. PhD Thesis, Department of Mechanical Engineering, University of Sheffield, 2011.

32. Gramacy RB. tgp: An R package for Bayesian nonstationary, semiparametric nonlinear regression and design by treed Gaussian process models. J Stat Softw 2007;19. doi:10.18637/jss.v019.i09.

33. Anonymous. EUROCOMP Design Code and Handbook, Structural Design of Polymer Composites. Edited by John L. Clarke, E \& FN SPON, 1996.

34. Anonymous. Fiberline design manual for structural profiles in composite materials. Fiberline Composites A/S, Kolding, Denmark, 1st Ed. 1995, updated 2nd Edition 2001.

35. CEN TC250 WG4L, Ascione L, Caron JF, Godonou P, Van Ijselmuijden K, Knippers J, Mottram JT, Oppe M, Gantriis Sorensen M, Taby J, Tromp L. Editors: Ascione L, Gutierrez E, Dimova S, Pinto A, Denton S. Prospect for New Guidance in the Design of FRP, Support to the implementation and further development of the Eurocodes, JRC Science and Policy Report JRC99714, EUR 27666 EN, European Union, Luxembourg, (2016), p 171 . ISBN 978-92-79https://amstat.tandfonline.com/doi/abs/10.1198/016214508000000689?casa_token=dpx4L8VN2XEAAAAA:8jh893Bmyc0 CDTkeJxcLS55_mEhAOypKcWhREMIDh9tp-a6dklnQQQEMKP3BCWbVHin6nqv_lrPijw225-1 doi:10.2788/22306.

36. EN ISO 14126:1999/AC:2002. Fibre-reinforced plastic composites - Determination of compressive properties in the inplane direction. 
37. EN ISO 527-4:1997. Plastics - Determination of tensile properties - Part 4: Test conditions for isotropic and orthotopic fibre-reinforced plastic composites.

38. EN 13706-1:2002 Reinforced plastic composites - Specification for pultruded profiles - Part 1: Designation; Part 2: Methods of test and general requirements; Part 3: Specification requirements

39. Juang JN, Pappa R. An eigensystem realization algorithm for modal parameter identification and model reduction. J Guid Control Dyn 1985;8(5):620-627.

40. Ewins DJ. Modal testing. Research Studies Press Ltd, 2000.

41. Cao Shancheng, Huajiang Ouyang. Robust multi-damage localisation using common eigenvector analysis and covariance matrix changes. Mechanical Systems and Signal Processing 111 (2018): 663-677. 
2020-08-28

Treed gaussian process for

\title{
manufacturing imperfection identification of pultruded GFRP thin-walled profile
}

\author{
Civera, Marco
}

Elsevier

Civera M, Boscato G, Zanotti Fragonara L. (2020) Treed gaussian process for manufacturing imperfection identification of pultruded GFRP thin-walled profile. Composite Structures, Volume 254, December 2020, Article number 122882

https://doi.org/10.1016/j.compstruct.2020.112882

Downloaded from Cranfield Library Services E-Repository 